\title{
CryoEM and Al reveal a structure of SARS-CoV-2 Nsp2, a multifunctional protein involved in key host processes
}

Kliment Verba ( $\boldsymbol{\nabla}$ kliment.verba@ucsf.edu )

University of California, San Francisco

Meghna Gupta

University of California, San Francisco

Caleigh Azumaya

University of California, San Francisco

Michelle Moritz

University of California, San Francisco

Sergei Pourmal

University of California, San Francisco

Amy Diallo

University of California, San Francisco

Gregory Merz

University of California, San Francisco

Gwendolyn Jang

University of California, San Francisco

Mehdi Bouhaddou

University of California, San Francisco

Andrea Fossati

University of California, San Francisco

Axel Brilot

University of California, San Francisco

Devan Diwanji

University of California, San Francisco

Evelyn Hernandez

University of California, San Francisco

Nadia Herrera

University of California, San Francisco

Huong Kratochvil

University of California, San Francisco

Victor Lam 
University of California, San Francisco

\section{Fei Li}

University of California, San Francisco

Yang Li

University of California, San Francisco

Henry Nguyen

University of California, San Francisco

Carlos Nowotny

University of California, San Francisco

\section{Tristan Owens}

University of California, San Francisco

\section{Jessica Peters}

University of California, San Francisco

\section{Alexandrea Rizo}

University of California, San Francisco

https://orcid.org/0000-0002-6142-8807

\section{Ursula Schulze-Gahmen}

The J. David Gladstone Institutes

\section{Amber Smith}

University of California, San Francisco

\section{Iris Young}

University of California, San Francisco

\section{Zanlin Yu}

University of California, San Francisco

\section{Daniel Asarnow}

University of California, San Francisco

\section{Christian Billesbølle}

University of California, San Francisco

\section{Melody Campbell}

Fred Hutchinson Cancer Research Center https://orcid.org/0000-0003-1909-5751

\section{Jen Chen}

University of California, San Francisco

\section{Kuei-Ho Chen}

University of California, San Francisco

\section{Un Seng Chio}

University of California, San Francisco

\section{Miles Dickinson}

University of California, San Francisco https://orcid.org/0000-0002-8016-1570

\section{Loan Doan}

University of California, San Francisco 


\section{Mingliang Jin}

University of California, San Francisco

\section{Kate Kim}

University of California, San Francisco

Junrui Li

University of California, San Francisco

\section{Yen-Li Li}

University of California, San Francisco

\section{Edmond Linossi}

The Walter and Eliza Hall Institute of Medical Research

\section{Yanxin Liu}

University of California, San Francisco https://orcid.org/0000-0002-2253-3698

\section{Megan Lo}

University of California, San Francisco

Jocelyne Lopez

University of California, San Francisco

Kyle Lopez

University of California, San Francisco

\section{Adamo Mancino}

University of California, San Francisco

Frank Moss III

University of California, San Francisco

Michael Paul

University of California, San Francisco

\section{Komal Pawar}

University of California, San Francisco

Adrian Pelin

University of California, San Francisco

Thomas Pospiech Jr.

University of California, San Francisco

\section{Cristina Puchades}

University of California, San Francisco

\section{Soumya Remesh}

University of California, San Francisco

\section{Maliheh Safari}

University of California, San Francisco

\section{Kaitlin Schaefer}

University of California, San Francisco

\section{Ming Sun}


Columbia University https://orcid.org/0000-0002-4413-2502

\section{Mariano Tabios}

University of California, San Francisco

\section{Aye Thwin}

University of California, San Francisco

\section{Erron Titus}

University of California, San Francisco https://orcid.org/0000-0001-6868-9121

\section{Raphael Trenker}

University of California, San Francisco

\section{Eric Tse}

University of California, San Francisco https://orcid.org/0000-0002-2082-9006

\section{Tsz Kin Martin Tsui}

University of California, San Francisco

\section{Feng Feng}

University of California, San Francisco

\section{Kaihua Zhang}

University of California, San Francisco

\section{Yang Zhang}

University of California, San Francisco

\section{Jianhua Zhao}

University of California, San Francisco

\section{Fengbo Zhou}

University of California, San Francisco

\section{Yuan Zhou}

University of California, San Francisco

\section{Lorena Zuliani-Alvarez}

University of California, San Francisco

\section{David Agard}

University of California, San Francisco https://orcid.org/0000-0003-3512-695X

\section{Yifan Cheng}

Howard Hughes Medical Institute https://orcid.org/0000-0001-9535-0369

\section{James Fraser}

University of California, San Francisco

\section{Natalia Jura}

University of California, San Francisco https://orcid.org/0000-0001-5129-641X

\section{Tanja Kortemme}

University of California, San Francisco https://orcid.org/0000-0002-8494-680X

\section{Aashish Manglik}

University of California, San Francisco https://orcid.org/0000-0002-7173-3741 


\section{Daniel Southworth}

University of California, San Francisco https://orcid.org/0000-0001-7108-9389

\section{Robert Stroud}

University of California, San Francisco https://orcid.org/0000-0003-2083-5665

\section{Danielle Swaney}

University of California, San Francisco

Nevan Krogan

University of California, San Francisco https://orcid.org/0000-0003-4902-337X

Adam Frost

University of California, San Francisco https://orcid.org/0000-0003-2231-2577

Oren Rosenberg

University of California, San Francisco https://orcid.org/0000-0002-5736-4388

\section{Article}

Keywords: SARS-CoV-2, proteins, drug design

Posted Date: May 19th, 2021

DOI: https://doi.org/10.21203/rs.3.rs-515215/v1

License: (a) (1) This work is licensed under a Creative Commons Attribution 4.0 International License. Read Full License 
Title: CryoEM and Al reveal a structure of SARS-CoV-2 Nsp2, a multifunctional protein involved in key host processes.

3

Authors: Meghna Gupta ${ }^{1 *}$, Caleigh M. Azumaya ${ }^{1 *}$, Michelle Moritz ${ }^{1 *}$, Sergei Pourmal ${ }^{1 *}$, Amy Diallo $^{1 *}$, Gregory E. Merz ${ }^{1 *}$, Gwendolyn Jang ${ }^{2,3,4,5 *}$, Mehdi Bouhaddou ${ }^{2,3,4,5 *}$, Andrea Fossati ${ }^{2,3,4,5 *}$, Axel F. Brilot', Devan Diwanji', Evelyn Hernandez', Nadia Herrera', Huong T. Kratochvil $^{1}$, Victor L. Lam ${ }^{1}$, Fei Li', Yang Li', Henry C. Nguyen ${ }^{1}$, Carlos Nowotny', Tristan W. Owens $^{1}$, Jessica K. Peters ${ }^{1}$, Alexandrea N. Rizo ${ }^{1}$, Ursula Schulze-Gahmen ${ }^{1}$, Amber M. Smith ${ }^{1}$, Iris D. Young ${ }^{1}$, Zanlin Yu', Daniel Asarnow ${ }^{1}$, Christian Billesbølle ${ }^{1}$, Melody G. Campbell ${ }^{1,6}$, Jen Chen ${ }^{1}$, Kuei-Ho Chen ${ }^{2,3,4,5}$, Un Seng Chio ${ }^{1}$, Miles Sasha Dickinson ${ }^{1}$, Loan Doan ${ }^{1}$, Mingliang Jin', Kate Kim ${ }^{1}$, Junrui Li', Yen-Li Li ${ }^{1}$, Edmond Linossi', Yanxin Liu', Megan Lo ${ }^{1}$, Jocelyne Lopez ${ }^{1}$, Kyle E. Lopez ${ }^{1}$, Adamo Mancino', Frank R. Moss III', Michael D. Paul', Komal Ishwar Pawar ${ }^{1}$, Adrian Pelin ${ }^{2,3,4,5}$, Thomas H. Pospiech Jr. ${ }^{1}$, Cristina Puchades ${ }^{1}$, Soumya Govinda Remesh ${ }^{1}$, Maliheh Safari ${ }^{1}$, Kaitlin Schaefer ${ }^{1}$, Ming Sun ${ }^{1,7}$, Mariano C Tabios ${ }^{1}$, Aye C. Thwin ${ }^{1}$, Erron W. Titus $^{1}$, Raphael Trenker ${ }^{1}$, Eric Tse ${ }^{1}$, Tsz Kin Martin Tsui ${ }^{1}$, Feng Wang ${ }^{1}$, Kaihua Zhang ${ }^{1}$, Yang Zhang $^{1}$, Jianhua Zhao', Fengbo Zhou', Yuan Zhou ${ }^{2,3,4,5}$, Lorena Zuliani-Alvarez ${ }^{1,2,3,4,5}$, QCRG Structural Biology Consortium ${ }^{1}$, David A Agard ${ }^{1,2,3,8}$, Yifan Cheng ${ }^{1,2,3,8,9}$, James $S$ Fraser $^{1,2,3,8}$, Natalia Jura ${ }^{1,2,3,11}$, Tanja Kortemme ${ }^{1,2,3,10,12}$, Aashish Manglik',2,3,13, Daniel R. Southworth ${ }^{1,2,3,8}$, Robert M Stroud ${ }^{1,2,3,8}$, Danielle L Swaney ${ }^{2,3,4,5}$, Nevan J Krogan ${ }^{2,3,4,5,16,8}$, Adam Frost ${ }^{1,2,3,8,14, \&}$, Oren S Rosenberg ${ }^{1,2,3,8,14,15,8}$, Kliment A Verba ${ }^{1,2,3,13, \&}$

\section{Affiliations}

${ }^{1} \mathrm{QBI}$ Coronavirus Research Group Structural Biology Consortium, University of California, San Francisco, CA 94158, USA.

${ }^{2}$ Quantitative Biosciences Institute (QBI) COVID-19 Research Group (QCRG), San Francisco, CA 94158, USA.

${ }^{3} \mathrm{QBI}$, University of California, San Francisco, CA 94158, USA.

${ }^{4}$ Department of Cellular and Molecular Pharmacology, University of California, San Francisco, CA 94158, USA.

5. David Gladstone Institutes, San Francisco, CA 94158, USA.

${ }^{6}$ Current affiliation: Division of Basic Sciences, Fred Hutchinson Cancer Research Center, Seattle, WA 98109, USA.

${ }^{7}$ Current affiliation: Beam Therapeutics, Cambridge, MA 02139, USA

${ }^{8}$ Department of Biochemistry and Biophysics, University of California, San Francisco, CA 94158, USA.

${ }^{9}$ Howard Hughes Medical Institute, San Francisco, CA 94158, USA.

${ }^{10}$ Department of Bioengineering and Therapeutic Sciences, University of California, San Francisco, CA 94158, USA.

${ }^{11}$ Cardiovascular Research Institute, University of California, San Francisco, CA 94158, USA.

${ }^{12}$ The University of California, Berkeley-University of California, San Francisco Graduate Program in Bioengineering, University of California, San Francisco, CA 94158, USA.

${ }^{13}$ Department of Pharmaceutical Chemistry, University of California, San Francisco, CA 94158, USA.

${ }^{14}$ Chan-Zuckerberg Biohub, San Francisco, CA 94158, USA.

${ }^{15}$ Department of Medicine, University of California, San Francisco, CA 94143, USA.

${ }^{16}$ Department of Microbiology, Icahn School of Medicine at Mount Sinai, New York, NY 10029, USA.

* These authors contributed equally to this work.

\& Corresponding authors. A.F., O.S.R. and K.A.V. are corresponding authors on behalf of QCRG Structural Biology Consortium 


\section{Abstract}

53 The SARS-CoV-2 protein Nsp2 has been implicated in a wide range of viral processes, but its

54 exact functions, and the structural basis of those functions, remain unknown. Here, we report an

55 atomic model for full-length Nsp2 obtained by combining cryo-electron microscopy with deep

56 learning-based structure prediction from AlphaFold2. The resulting structure reveals a

57 highly-conserved zinc ion-binding site, suggesting a role for Nsp2 in RNA binding. Mapping

58 emerging mutations from variants of SARS-CoV-2 on the resulting structure shows potential

59 host-Nsp2 interaction regions. Using structural analysis together with affinity tagged purification

60 mass spectrometry experiments, we identify Nsp2 mutants that are unable to interact with the

61 actin-nucleation-promoting WASH protein complex or with GIGYF2, an inhibitor of translation

62 initiation and modulator of ribosome-associated quality control. Our work suggests a potential

63 role of $\mathrm{Nsp} 2$ in linking viral transcription within the viral replication-transcription complexes

64 (RTC) to the translation initiation of the viral message. Collectively, the structure reported here,

65 combined with mutant interaction mapping, provides a foundation for functional studies of this

66 evolutionary conserved coronavirus protein and may assist future drug design.

\section{Introduction}

Upon entry into human cells SARS-CoV-2, the causative agent of COVID-19, produces

70 two large polyproteins, pp1a and pp1ab. These polyproteins are further processed by two viral

71 proteases into 16 individual non-structural proteins (nsp1-nsp16). These non-structural proteins

72 fulfill a number of essential viral functions including RNA replication, replication proofreading,

73 double-membrane vesicle formation, and others ${ }^{1}$. Many of these also interact with host factors

74 to effectively subvert the host cell to meet the virus's needs ${ }^{2}$. Such subversion, for example,

75 includes suppressing host innate immune responses, host translation, nuclear import, and other

76 effects $^{3-5}$. Despite their central importance to viral pathogenesis, many non-structural proteins

77 remain structurally and functionally uncharacterized. Furthermore, the interactions between 
78 virus and host proteins are even less understood, with only a handful of unique viral-host protein

79 complex structures available. As viruses often hijack central nodes in host cell pathways,

80 studying viral-host interactions in molecular detail can lead to a better understanding of the

81 mechanisms of viral pathogenesis and of the fundamental host cell processes the virus targets.

82 In addition, viral-host protein complexes are an attractive target for antiviral therapeutics as they

83 are less likely to accrue resistance. In this report we focus on one of the least studied

84 SARS-CoV-2 proteins, Nsp2.

85 Neither the functions nor the structure of Nsp2 are known. In SARS-CoV-1, Nsp2

86 deletion leads to a defect in viral replication but still yields viable viruses ${ }^{6}$. Interestingly,

87 expression of Nsp2 from an alternate site in the genome does not rescue this defect, likely

88 indicating the importance of correct timing for Nsp2 expression ${ }^{7}$. A number of studies mapping

89 host-viral interactomes of SARS-CoV-1/2 and MERS have identified host proteins that interact

90 with $\mathrm{Nsp}^{8-11}$. These studies have implicated Nsp2 in processes ranging from translation

91 repression to endosomal transport, ribosome biogenesis, and actin filament binding. Nsp2 in

92 SARS-CoV-2 and other coronaviruses have been observed to localize to endosomes and

93 replication-transcription complexes (RTC); but it's currently unclear what role Nsp2 plays at

94 these sites ${ }^{10,12}$. Although Nsp2 is present in SARS-CoV-1, CoV-2, MERS-and in closely related

95 coronaviruses in bats, pangolins and other animals - there is a considerable sequence variation

96 across different species (Sup Fig 1). This degree of variability may indicate rapid Nsp2

97 adaptation under host-specific selection pressures. Furthermore, genome sequencing of

98 SARS-CoV-2 variants during the COVID19 pandemic reveals sites under positive selection in

99 Nsp2, suggesting host-specific human adaptation following successful zoonotic transfer ${ }^{13}$.

100 Importantly, a specific mutation in Nsp2, T85I, is observed in clade 20C (as per Nexstrain

101 nomenclature) including both variants of interest (B.1.526) and variants of concern

102 (B.1.427/B.1.429 recently identified in California) $)^{14,15}$. Genetic knockdown/knockout studies have 
shown that a number of Nsp2 host interactors negatively affect SARS-CoV-2 replication, further

104 corroborating the functional importance of Nsp2 ${ }^{10,16}$.

105 Structural models of Nsp2 and Nsp2-host protein complexes will allow spatial mapping 106 of the growing list of mutations and potentially shine light on their significance for Nsp2

107 functions. By delineating which Nsp2 surfaces determine specific Nsp2-host protein interactions,

108 researchers will be able to generate specific Nsp2 point mutants that are defective in forming

109 these protein complexes-helping tease apart the biological role of a particular Nsp2-host

110 interaction. However, there are neither structures available for Nsp2 from any of the beta

111 coronaviruses family members nor are there any similar structures in the PDB based on the

112 sequence homology. Here we present a structure of SARS-CoV-2 Nsp2 derived from a

113 combination of cryo-EM experimental data and AlphaFold2 prediction. Utilizing the natural

114 sequence variation in SARS-CoV-2 together with our structure and mass spectrometry

115 experiments, we identify two key Nsp2 surfaces that are required for specific host interactions.

117 Results

119 Combining a cryo-EM map with AlphaFold2 Nsp2 predictions yields a pseudo-atomic 120 structure for full-length Nsp2 Full-length SARS-CoV-2 Nsp2 was recombinantly expressed and purified from E.coli cells. After purification involving multiple steps to remove contaminating nucleic acids, Nsp2 was obtained at high purity, plunge frozen, and imaged by cryo-electron microscopy. Processing in

124 cryoSPARC2 $2^{17,18}$ followed by RELION3 ${ }^{19}$ yielded a reconstruction of Nsp2 at $3.8 \AA$ global

125 resolution ranging from $3 \AA$ in the best resolved regions to $6 \AA$ in the most flexible regions (Fig $1 \mathrm{~A}$

126 and Sup Fig 2). The initial model was automatically built into the well-resolved region using the

127 DeepTracer ${ }^{20,21}$ online server and then further corrected and refined via manual manipulation in

128 Coot $^{22}$ and ISOLDE ${ }^{23}$, followed by final refinements in Phenix ${ }^{24}$ and Rosetta ${ }^{25}$ (see methods). 
129 Having built an initial model, we then identified a number of putative zinc binding sites and

130 repeated the sample purification and cryo-EM imaging in the presence of zinc. This yielded an

131 improved 3.2 A cryo-EM map, which revealed additional details and enabled improved modeling

132 of residues 5-505 of the SARS-CoV-2 Nsp2 (Fig 1B). To our surprise, under these Zn-included

133 conditions the density for the C-terminal 130 amino acids was completely missing. In the

134 cryo-EM map without zinc, although the density for the flexible C-terminal domain was present,

135 it was resolved at between 5-6 $\AA$ resolution. The closest homologous structure showed less

136 than $10 \%$ sequence identity $(\mathrm{PDB}: 3 \mathrm{LD} 1)^{26}$, and as the domain was predicted to be high in the

137 beta sheet fold this posed a significant challenge for de novo modeling based on low-resolution

138 cryo-EM density alone.

139 The recent utilization of deep learning for protein structure prediction based on amino

140 acid sequence has led to a new level of success, as demonstrated by CASP $14^{27}$. Specifically,

141 the AlphaFold2 team was able to predict protein structures with unprecedented accuracy,

142 producing results sometimes indistinguishable from the experimentally derived structures.

143 AlphaFold2 and other teams in the CASP14 also ran predictions on SARS-CoV-2 proteins,

144 including Nsp2. Out of all the available predicted models for Nsp2, only one model has an

145 RMSD of less than $20 \AA$ to our experimental model: C1901TS156_4 from the AlphaFold2 team.

146 The other 5 models from AlphaFold2 were also close to $20 \AA$ RMSD, so we aligned all the

147 available Nsp2 models from the AlphaFold2 team (5 from the CASP14 and one updated model

148 available on their website ${ }^{28}$ ) to our structure (Sup Fig 3). This comparison made it clear that,

149 globally, the predictions were quite different from the experimentally derived structure. In

150 addition, the most updated model was missing a prediction for 93 amino acids of the protein (Fig 151 1C).

152 However, when analyzed in isolation, the individual motifs and domains of the proteins

153 are remarkably close to the experimentally derived structure. This observation prompted us to

154 break the model down into 4 subregions and align them to the experimentally derived structure 
155 independently. This yielded high local similarity per domain (average RMSD values of less than

$1562 \AA$, Fig 1D). The prediction for the missing C-terminal 130 amino acids in isolation fit well within

157 the lower resolution density for that domain in the cryoEM map without zinc. We therefore

158 combined the AlphaFold2 domain prediction for the C-terminal 130 amino acids with our

159 experimentally built cryo-EM model to yield an experimentally valid and complete structure of 160 full-length Nsp2 (Fig 1E, Sup Table 1).

161

162 Nsp2 shows low global conservation among beta-coronaviruses, but possesses a highly conserved Zn binding motif.

164 To better understand which regions of Nsp2 are functionally important, we performed a 165 sequence alignment of Nsp2 across beta-coronaviruses from different species. Nsp2 shows low 166 conservation, with the $\mathrm{N}$-terminal half of the protein being marginally more conserved (Fig 2A 167 and Sup Fig 1). Overall, SARS-CoV-2 Nsp2 is 68\% identical to SARS-CoV-1 Nsp2 and only $16820 \%$ identical to MERS virus Nsp2. Strikingly, the most conserved residues are a cysteine quad 169 coordinating a $\mathrm{Zn}^{2+}$ ion in a $\mathrm{Zn}$ ribbon like motif, with three of the four cysteines being invariant 170 across all the virus sequences. Performing a structural similarity search with this motif from 171 Nsp2 indicates that it is similar to zinc ribbons ${ }^{29}$ in a number of RNA binding proteins in RNA 172 polymerases and ribosomes (Fig $2 A$ insert, average RMSD for the region of $1.7 \AA$ ). In some of 173 these proteins these motifs explicitly have been implicated in RNA binding and in one structure 174 (PDB:1JJ2, chain 2), the zinc ribbon on the ribosomal protein L44E is directly interacting with 175 the ribosomal RNA. This motif is also similar to the tudor domains in the histone tail binding 176 protein JMJD2A (RMSD of $1.7 \AA$ ). Although the fold is similar, the tudor domain is missing a $177 \mathrm{Zn}^{2+}$ ion in the JMJD2A structure (PDB:2QQS). Previous studies have associated Nsp2 with the 178 viral $\mathrm{RTCs}^{12,30}$ and during the purification from bacteria we observed strong, apparently 179 non-specific binding to E.coli nucleic acids that required chromatographic separation. One 180 possibility, therefore, is that this motif is important for Nsp2 interactions with nucleic acids. 
In addition to the proteins containing zinc ribbons and tudor motifs, a search of the PDB

182 for structurally similar proteins returned only one additional structure, the structure of Nsp2 from

183 Avian Infectious Bronchitis virus (PDB:3LD1). Although the sequence identity is below $10 \%$ for

184 these proteins, the beta sheet C-terminal domain aligns well with our model. No other structures

185 came up in our structural similarity search with either the FATCAT ${ }^{31}$ or DALI ${ }^{32}$ servers.

186

187 Subsets of acquired mutations in Nsp2 group into surface patches.

188 Examining the mutations that occur in SARS-CoV-2 Nsp2 during the COVID19

189 pandemic, over 50 sites have been identified as being under positive selection (at the time of

190 writing, based on the $\mathrm{dn} / \mathrm{ds}>1$ metric $\left.^{33,13}\right)$. Most of these mutations occur at low frequency. Two

191 mutations however, T85I and I120F, are present at frequencies of roughly $13 \%$ and $5 \%$

192 respectively. The T85I mutation maps to a surface residue on our structure (Fig 3). The side

193 chain of T85 is surface exposed, therefore replacing it with a hydrophobic isoleucine should not

194 be favorable. However, if this region of Nsp2 is involved in protein-protein interactions such a

195 substitution might be a gain-of-function change, stabilizing a hydrophobic binding interface. The

196 second residue that is mutated, I120F, is not surface exposed and instead packs in a

197 hydrophobic core that anchors a small helix. This small helix is attached to a highly charged

198 loop on the surface of the protein and its dynamics may be functionally important. A

199 phenylalanine mutation may further stabilize this helix anchor point by participating in

200 pi-stacking interactions with neighboring aromatic residues (Fig 3 inset).

201 The structure allowed us to map the spatial relationships of conserved residues in Nsp2

202 among SARS-CoV-2 strains, revealing unexpected regions of conservation and selection. To

203 identify rapidly evolving regions of the protein, we mapped all the positively-selected mutations

204 to the protein surface (Fig 4). This analysis revealed charged surfaces which are devoid of

205 mutations, potentially indicating surfaces important for conserved interactions (Fig 4). There are

206 also two residue clusters where mutations found in strain variants are proximal to one another 
207 and alter the characteristics of the protein's surface in similar ways. Cluster 1 is near the

$208 \mathrm{~N}$-terminus consisting of three arginine residues (R27C, R52C, R4C) that mutate individually to 209 cysteines, reducing the exposed positive surface charge in that region and introducing a 210 sulfhydryl. Cluster 2 consists of six proximal residues which mutate individually to more

211 hydrophobic residues (G262V, G265V, G285V, A411V, T371I) (Fig 4) in the variant strains. In

212 both clusters, only individual single residue mutations are picked up in the viral population but

213 the fact that they form physical clusters and have similar biochemical consequences might 214 indicate adaptation.

215

216 Affinity purification mass spectrometry identifies Nsp2 surfaces mediating specific host

217 interactions.

218 To investigate whether common strain variants as well as disruptions of conserved

219 patches would affect Nsp2's ability to interact with host proteins we generated appropriate Nsp2

220 mutants and assessed changes in virus-human protein-protein interactions by affinity

221 purification mass spectrometry (AP-MS) in HEK293T cells ${ }^{2}$. Three Nsp2 mutants were based on

222 the natural Nsp2 variations: T85I, D23Y/R27C (cluster 1), G262V/G265V (cluster 2) and two

223 were designed to disrupt the conserved/charged surface patches: K330D/K337D and

224 E63K/E66K. All mutants expressed at similar levels in HEK293T cells (Sup Fig 4). We began by

225 performing a global and unbiased quantification of virus-host protein-protein interactions using

226 an automated affinity purification mass spectrometry workflow (see Methods). T85I and

227 D23Y/R27C did not show significant changes in their interactomes, but three mutants did show

228 significant changes in host interactions: G262V/G265V mutations abrogated Nsp2 interactions

229 with the complex comprising GIGYF2/EIF4E2/RNF59834, E63K/E66K reduced interactions

230 between Nsp2 and WASHC4/WASHC5 complex and also FKBP15, and K330D/K337D had a

231 severely reduced interaction with NADPH cytochrome P450 reductase (POR gene) (Figure

232 5A,B,D, Table S2). Interestingly, Nsp2 E63K/E66K also gained a large number of new 
233 interactors which are predominantly involved in ribosomal RNA metabolic processes (Sup Fig

234 5). To increase the sensitivity and robustness of our quantitation, we performed Parallel

235 Reaction Monitoring (PRM) on the subset of significantly changed interactors from the Data

236 Dependent Analysis (DDA). Overall the PRM analysis recapitulated the findings of the DDA

237 (Figure 5C).

238

239 Discussion

240 In this report, we were able to combine cryoEM with recent advances in de novo protein

241 predictions to obtain a complete atomic model for SARS-CoV-2 Nsp2 protein. Although there

242 was a recent report of using AlphaFold2 predicted protein structure of Orf8 to solve the phase

243 problem in crystallographic studies, to our knowledge this is the first explicit use of AlphaFold2

244 predictions with restraints from an experimental cryoEM density for model building ${ }^{35}$. This

245 exercise suggests that domain structure predictions from deep neural networks are increasingly

246 likely to be locally accurate and, when combined with experimental restraints, sufficient for

247 global structure prediction and integrative structural modelling. Electron cryo-microscopy and

248 cryo-tomography will be important sources of such overall shape information, and readily

249 obtainable, low-resolution measurements like negative stain electron microscopy, small-angle

250 X-ray scattering, cross-linking mass spectrometry, or even biochemical experiments may

251 provide sufficient constraints for accurate, global models to be determined in combination with

252 predicted domain structures. It is possible that further improvements in the prediction algorithms

253 will eliminate the need for experimental measurements entirely. However, atomic resolution

254 structures of multi-component and multi-domain assemblies are still relatively uncommon, and

255 this deficit of appropriate training data in the PDB may limit the accuracy of computational

256 models for multi-domain assemblies and higher-order complexes. Put another way, the

257 deficiency of data about protein-protein interfaces may mean that de-novo predictions of

258 complex assemblies will remain underdetermined for some time. Future work will explore the 
use of restraints from 3D cryo-EM maps, 2D images, tomograms, and other data sources like

260

262 the Nsp2 of SARS-CoV-2 suggests a number of biological roles for Nsp2 and also regions of

263 interest on the protein. We identify a highly conserved zinc ribbon motif which structurally is

264 highly similar to zinc ribbons in RNA binding proteins. One possibility, therefore, is that this motif

265 is important for Nsp2 interactions with nucleic acids. Interestingly our mass spectrometry studies

266 on the E63K/E66K mutant, designed to introduce a charge reversal mutation in a conserved

267 negatively charged surface patch (Figs 4,5 and Sup Fig 5), show that this mutant gains a large

268 number of partners involved in ribosome biogenesis. Concurrently with this gain, this mutant

269 loses interactions with the endosomal/actin machinery (FKBP15, WASHC proteins ${ }^{36,37}$ ). It is

270 tempting to speculate that Nsp2 binds ribosomal RNA (via the identified zinc ribbon) but

271 attachment to cytoskeletal elements at endosomes limits its interactions with rRNA. Upon

272 disruption of the endosomal anchoring of Nsp2 by the above mutations, Nsp2 is free to interact

273 with ribosomal RNAs-potentially even translocating to the nucleolus. Interestingly, previous

274 proximity labeling studies on murine coronavirus Nsp2 have uncovered an exciting link between

275 the viral polymerase within RTCs and the host cell's translation machinery ${ }^{12}$. Since Nsp2 has

276 affinity for ribosomal RNA and is localized to RTCs, one appealing model is that Nsp2 plays a

277 role in locally enriching ribosomes next to viral messages for more efficient

278 transcriptional-translational coupling in the cytosol. Another aspect of these specific interactions

279 are potential roles that Nsp2 may play in hijacking the endosomal pathway to meet viral needs.

280 WASH complexes have been shown to play key roles in exocytosis and endosome biogenesis,

281 including cargo sorting through local Arp2/3 complex activation ${ }^{38}$. SARS-CoV-2 enters cells

282 through the endosomal pathway and it may be functionally important for the virus to modulate

283 endosome pathways for successful infection. 
285 in modulating ribosome-associated quality control. This mutant is based on the natural variants 286 of Nsp2 in the patch that is becoming more hydrophobic during the 2019-2020 COVID19 287 pandemic (Figure 4, cluster 2), although the natural variants all have a single mutation, either 288 the G262V or G265V. This mutant is the only one that specifically loses interactions with 289 GIGYF2, EIF4E2 and RNF598. These three proteins are known to form a complex and have 290 been implicated in inhibiting translation initiation when ribosomes stall on defective or abnormal 291 mRNA messages ${ }^{34}$. At the moment it is unclear what is the exact functional connection between 292 SARS-CoV-2 infection and this recently discovered component of ribosome-associated quality 293 control mediated by GIGYF2/EIF4E2. Given that SARS-CoV-2 has optimized codon usage for 294 the human host, it is unlikely that there is increased ribosomal stalling on the viral message that 295 requires inhibition of GIGYF2/EIF4E2 by Nsp2 ${ }^{39,40}$. Furthermore, genetic studies have shown 296 that GIGYF2 and EIF4E2 are necessary rather than inhibitory for viral replication ${ }^{16}$. Perhaps the 297 virus uses Nsp2 to inhibit translation initiation of host messages, freeing ribosomes and the rest 298 of the translation machinery for virus production. Indeed, a prior study of the 299 GIGYF2/EIF4E2/ZNF598 complex suggests that in addition to its role in RQC, this complex 300 selectively suppresses transcripts involved in host inflammatory signaling, including IL- $8^{41}$. It is 301 worth noting that Nsp2 interactions with GIGYF2/EIF4E2/ZNF598 complex is conserved across 302 SARS-CoV-1 and MERS indicating that this interaction might be of therapeutic importance for 303 coronaviruses generally ${ }^{10}$.

304 Our mass spectrometry experiments of the most prevalent Nsp2 mutation, T85I, did not 305 identify any changes in host interactions of this mutant. This may be due to our experiments 306 lacking the context of other viral proteins that would be present in a bona fide infection or 307 potentially due to the wrong cellular context. Alternatively this may suggest that some mutations 308 do not confer any fitness benefit and are simply present due to the C-U hypermutation observed 309 in SARS-CoV-2, which is likely driven by host mediated APOBEC editing ${ }^{42}$. Interestingly, there is 
310 a recent report demonstrating that the SARS-CoV-2 Nsp2 T85I mutant shows a minor

311 replication defect in Vero green monkey cells, but has no effect in human cells. This is

312 consistent with the T85I mutation not conferring a strong selective advantage ${ }^{43}$. Globally, the

313 second most prevalent SARS-CoV-2 amino acid substitution that is driven by the C-U

314 hypermutation is a $\mathrm{T}$ to I change. Therefore the T85I mutation in the 20C clade of SARS-CoV-2

315 may be neutral in fitness, but stable due to host-mediated RNA editing.

316 Overall, analysis of the resulting Nsp2 structure revealed a rapidly evolving protein

317 surface, with potential consequences for host-virus interactions. Leveraging the new structure

318 with natural Nsp2 variations and mass spectrometry we were able to identify surfaces important

319 for specific Nsp2 interactions. The pattern of disruption of interactions points to at least three

320 specific areas of biology that Nsp2 is involved in: interactions with endosomes through

321 cytoskeletal elements, interactions with modulators of translation, and also direct interactions

322 with ribosomal RNA. The exact roles Nsp2 plays in these pathways will require further

323 experimental characterization using the structure-based point mutants described here.

\section{Methods}

\section{Nsp2 Expression}

328 SARS-CoV-2 Nsp2, codon optimized for Escherichia coli expression, was cloned in a 329 pET-29b(+) vector backbone with N-terminus 10XHis-tag and SUMO-tag (Ep156). For 330 expression, the plasmid Ep156 was transformed in the LOBSTR E. coli strain and a single 331 colony was inoculated in LB media with $50 \mu \mathrm{g} / \mathrm{ml}$ kanamycin overnight at $37^{\circ} \mathrm{C} .1 \%$ overnight 332 culture was inoculated in $1 \mathrm{~L}$ TB media with $50 \mu \mathrm{g} / \mathrm{ml}$ kanamycin and and grown at $37^{\circ} \mathrm{C}$ till 333 O.D. $600 \mathrm{~nm}$ reached 0.8 . The culture was transferred to $20^{\circ} \mathrm{C}$ and induced with $0.5 \mathrm{mM}$ IPTG for $33416 \mathrm{~h}$. The cells were harvested and washed with PBS, flash-frozen and stored at $-80^{\circ} \mathrm{C}$. 


\section{Nsp2 Purification}

337 To a $6 \mathrm{~L}$ equivalent of cell pellet, $150 \mathrm{~mL}$ of lysis buffer $(50 \mathrm{mM}$ HEPES pH 7.5, $300 \mathrm{mM} \mathrm{NaCl}$,

$33810 \%$ glycerol, $2 \mathrm{mM} \mathrm{MgCl}_{2}$ ) supplemented with 2 protease inhibitor tablets (Roche), $1 \mathrm{mM}$

339 PMSF, and $30 \mu \mathrm{L}$ benzonase nuclease (Millipore Sigma) was added. Cells were resuspended

340 and dounce homogenized before either sonication ( $3 \times 10$ min cycles using a sonifier (Branson),

341 at $50 \%$ duty cycle ( $1 \mathrm{sec}$ on, $1 \mathrm{sec}$ off), followed by $\geq 5 \mathrm{mins}$ on ice, or high pressure

342 homogenizer ( 3 times passage through the EmulsiFlex-C3 [Avestin] at $\sim 15,000$ psi, $4^{\circ} \mathrm{C}$ ). After

343 centrifugation for 40 mins at $25,000 \times x g, 4^{\circ} \mathrm{C}$, clarified samples were transferred to a $50 \mathrm{~mL}$

344 conical tube and supplemented with a final $20 \mathrm{mM}$ imidazole $\mathrm{pH} 7.5$ before batch-binding with

$345 \mathrm{Ni}-\mathrm{NTA}$ superflow resin (Qiagen) for $\sim 1 \mathrm{hr}$ at $4^{\circ} \mathrm{C}$. The resins were collected in a gravity flow

346 column, washed with 15 CV lysis buffer, followed by 2x 7.5 CV sulfate wash buffer (25 mM Tris

$347 \mathrm{pH} 8.5,300 \mathrm{mM} \mathrm{NaCl}, 10 \%$ glycerol, $\left.100 \mathrm{mM} \mathrm{Na}_{2} \mathrm{SO}_{4}\right), 2 x 7.5 \mathrm{CV}$ wash buffer (25 mM Tris pH

348 8.5, $300 \mathrm{mM} \mathrm{NaCl}, 10 \%$ glycerol, $30 \mathrm{mM}$ imidazole), 2x $7.5 \mathrm{CV}$ wash buffer supplemented with

$3492 \mathrm{mM} \mathrm{ATP}, 4 \mathrm{mM} \mathrm{MgCl}$, and eluted in 2x $2.5 \mathrm{CV}$ elution buffer (25 mM Tris pH 8.0, $300 \mathrm{mM}$

$350 \mathrm{NaCl}, 10 \%$ glycerol, $300 \mathrm{mM}$ imidazole, $2 \mathrm{mM} \mathrm{MgCl}$ ). The elution was treated with benzonase

351 and Ulp1 protease and dialyzed overnight at $4^{\circ} \mathrm{C}$ in dialysis buffer $(25 \mathrm{mM}$ Tris $\mathrm{pH} 8.5,75 \mathrm{mM}$

$352 \mathrm{NaCl}, 10 \%$ glycerol, $0.5 \mathrm{mM}$ TCEP, $2 \mathrm{mM} \mathrm{MgCl}_{2}$ ). The tagless $\mathrm{Nsp} 2$ was further purified using a

$3535 \mathrm{~mL}$ HiTrap Heparin HP column (Cytiva) using a linear gradient of 7.5\% Heparin buffer A (30

$354 \mathrm{mM}$ Tris $\mathrm{pH} 8,1 \mathrm{mM}$ DTT) to $50 \%$ Heparin buffer $\mathrm{B}$ (30 mM Tris pH 8, $1 \mathrm{M} \mathrm{NaCl}, 1 \mathrm{mM}$ DTT).

355 Peak fractions corresponding to Nsp2 were concentrated and further purified using a Superdex

356200 increase 10/300 GL column (Cytiva) in SEC buffer (20 mM Tris pH 8.0, $250 \mathrm{mM} \mathrm{NaCl}, 0.5$

$357 \mathrm{mM}$ TCEP) to yield a single peak. The peak fractions were pooled and concentrated and used

358 for CryoEM. For the $\mathrm{Zn}$ containing sample, $10 \mu \mathrm{M} \mathrm{ZnCl}$ was kept in all the buffers of the 359 purification protocol mentioned above.

360

361 CryoEM grid freezing and data collection 
362 Purified nsp2 was diluted to $6 \mu \mathrm{M}$ for the apo sample and $5.7 \mu \mathrm{M}$ for the $\mathrm{Zn}$ containing sample.

363400 mesh 1.2/1.3R Au Quantifoil grids were glow discharged at $15 \mathrm{~mA}$ for 30 seconds.

364 Vitrification was done using FEl Vitrobot Mark IV (ThermoFisher) set up at $4^{\circ} \mathrm{C}$ and $100 \%$

365 humidity. $3.5 \mu \mathrm{l}$ sample was applied to the grids and the blotting was performed with a blot force

366 of 0 for $4-6 \mathrm{~s}$ prior to plunge freezing into liquid ethane. For the apo sample, two datasets

367 comprising of 804 and 1116 118-frame super-resolution movies each were collected with a $3 \times 3$

368 image shift at a magnification of $105,000 x$ with physical pixel size of $0.834 \AA$ pix on a Titan Krios

369 (ThermoFisher) equipped with a K3 camera and a Bioquantum energy filter (Gatan) set to a slit

370 width of $20 \mathrm{eV}$. Collection dose rate was $8 \mathrm{e}^{-/ / p i x e l / s e c o n d ~ f o r ~ a ~ t o t a l ~ d o s e ~ o f ~} 66 \mathrm{e}^{-} / \AA^{2}$. Defocus

371 range was 0.8 to $2.4 \mu \mathrm{m}$. Each collection was performed with semi-automated scripts in

372 SerialEM. Nsp2 with Zn grids were prepared using a similar protocol. 1149 118-frame movies

373 were collected for this sample at a $105,000 x$ magnification with physical pixel size of $0.834 \AA /$ pix

374 on Titan Krios similar to without Zn sample. Collection dose rate was 8 e/pixel/second for a total

375 dose of $67 \mathrm{e}^{-/} / \AA^{2}$.

376

377 Data Processing

378 Without Zn: Initial processing was done in Cryosparc (v2.15.0) ${ }^{1718}$. The first dataset with 801 379 dose-weighted motion corrected micrographs ${ }^{44}$ was imported and Patch CTF(M) was 380 performed. This dataset required thorough manual curation and led to selection of 388 381 micrographs for further processing. Blob-picker was used to pick 363145 particles and 382 extraction was done with a box size of 288 px. 2D-classification was done into 150 classes and 383 good looking classes were selected with total 91181 particles. A second dataset with 1116 384 dose-weighted micrographs was processed in a similar manner. After Patch CTF(M), 748 385 micrographs were curated based on CTF-fit resolution $(<5 \AA)$, ice-thickness, and carbon. 386 Templates were created from the previous dataset and used for template-based particle picking 387 to get 577518 particles. 240551 particles were selected after 2D-classification into 200 classes. 
388 These particles were merged with 91181 selected particles of the previous dataset after 389 2D-classification. Total 331732 particles were classified into 3 classes with heterogeneous 390 refinement. Multiple rounds of heterogeneous refinements, non-uniform refinements and 391 homogenous refinements resulted in a $3.45 \AA$ 3D-reconstruction with 99076 particles. The 392 particles from this final step of Cryosparc processing were imported into Relion ${ }^{19}$ (version 3.0.8) 393 for further processing. 3D-classification without mask resulted in a $3.59 \AA$ map. The core of the 394 map was better resolved therefore skip-align classification was done on the core. The best class 395 was subjected to 3D-refinement and post-processing leading to the final map at $3.49 \AA$. This 396 class was selected and skip-align classification was done for the full map. The overall resolution 397 of the full map with the selected 42579 particles was reported to be $3.76 \AA$ which upon manual 398 inspection was the best looking map even though nominally being at worse resolution than 399 some previous reconstructions.

400

401 With Zn: Like the 'without Zn' dataset, for initial processing we used Cryosparc (v2.15.0). Patch 402 CTF(M) was performed on imported 1149 dose-weighted micrographs. The micrographs were 403 curated for CTF-fit resolution of better than $5 \AA$. Template-based particle picking was done 404 (templates from the without $\mathrm{Zn}$ dataset) on the selected 1028 micrographs, resulting in 1515264 405 particles. A series of ab-initio classifications followed by heterogeneous refinements and 406 non-uniform refinements on the best classes selected led to a map of $3.1 \AA$ with 81817 407 particles. These particles were transferred to Relion (version 3.0.8) and a single-class skip-align 408 classification was performed with a mask. A 3.15 A map with 81817 particles was obtained after 409 3D-refinement and post-processing on the particles from skip-align classification.

\section{Refinement/Model building}

412 The initial model for the core of 'without Zn' map was initially obtained by submitting the high 413 resolution region of the map with the full Nsp2 sequence to the DeepTracer server ${ }^{2021}$. This 
414 resulted in two chains which threaded the backbone fairly well but contained amino acid 415 substitutions/deletions. Therefore a homology model was built for Nsp2 using the resulting 416 model from DeepTrace server as a template, using SwissModel server. This model was then 417 refined against the map in Phenix Real Space Refine ${ }^{24}$ and iteratively rebuilt with Rosetta 418 (2020.08 release $)^{25}$. Best scoring models were manually examined and corrected using COOT $4190.9^{22}$. Rosetta was used for automatic iterative rebuilding the lower resolution regions of the map 420 and loops (212-224, 475-490). Cys/His residues were manually identified for Zn coordination

421 sites and $\mathrm{Zn}$ was placed in COOT. At this point the higher resolution cryo-EM map obtained in 422 the presence of zinc was used for downstream steps. Map/model quality was examined and 423 ramachandran outliers were fixed in ISOLDE $1.0^{23}$. Rosetta FastRelax was used in cartesian 424 space followed by iterations of refinement in Phenix Real Space Refine. This fixed most of the 425 geometry outliers but introduced a large number of clashes in the model. Rosetta FastRelax in 426 torsion space was used on the model from Phenix Real Space Refine to resolve the clashes 427 while preserving good model statistics for the final model. The b-factors were assigned using 428 Rosetta B-factor fitting mover. Local resolution was determined by running ResMap program ${ }^{45}$. 429 Directional FSC curves were determined by submitting the associated files to the 3DFSC 430 server $^{46}$.

431

\section{Obtaining full Nsp2 model incorporating AlphaFold2 prediction}

433 Predicted Nsp2 models were downloaded either from the DeepMind website or CASP14 434 website and then were aligned to the experimental Nsp2 map using matchmaker tool in 435 Chimera ${ }^{47,48}$. Based on visual examination, the most updated (at the time of writing) 436 AlphaFold2 model (V3_4_8_2020) was split into 4 domains: 1-277, 278-344, 459-509 and 437 510-638. First three were individually aligned with matchmaker in ChimeraX to the experimental 438 model to assess the similarity and report RMSDs in the main text. These regions were not used 439 for downstream model building. The fourth region, 510-638 was rigid body fit into the $3.8 \AA$ 
cryo-EM map (obtained without zinc). The experimental model was then stitched together with

441 the rigid body fit domain for residues 510-638 from AlphaFold2. The whole model was energy

442 minimized into the cryo-EM density filtered to $5 \AA$ by running Rosetta FastRelax in torsion

443 space.

444

\section{Sequence alignment and sequence conservation analysis}

446 Nsp2 sequences were manually downloaded from UniProt and aligned in Jalview using

447 MAFFT $^{49,50}$. Conservation was mapped on the Nsp2 structure using a combination of Chimera

448 and ChimeraX and the supplementary alignment figure was prepared with MView server ${ }^{47,51,52}$.

\section{9}

450 SARS-CoV-2 Nsp2 mammalian expression constructs. SARS-CoV-2 isolate 451 2019-nCoV/USA-WA1/2020 (accession MN985325), an early-lineage sequence downloaded on 452 January 24, 2020, was the reference sequence for all viral expression constructs. Native 453 nucleotide sequences encoding proteolytically mature Nsp2 were first codon optimized 454 (https://www.idtdna.com/codonopt) for gene synthesis. The gBlock Gene Fragment (IDT) 455 encoding Nsp2 and the C-terminal linker and 2x-Strep tag was inserted into 456 pLVX-EF1alpha-IRES-Puro at the EcoRI and BamHI restriction sites by In-fusion cloning [PMID: 457 32353859]. SARS-CoV-2 Nsp2 mutants (D23Y/R27C, E63K/E66K, T85I, G262V/G265V, and $458 \mathrm{~K} 330 \mathrm{D} / \mathrm{K} 337 \mathrm{D}$ ) were generated in a similar manner using unique restriction sites within the 459 Nsp2 sequence to excise segments containing wild type residues. Sequences were 460 subsequently replaced by In-fusion cloning with gBlocks (IDT) containing mutated residues. All 461 mutations were confirmed by sequencing.

462

463 Cell culture. HEK293T cells were cultured in Dulbecco's Modified Eagle's Medium (Corning or 464 Gibco, Life Technologies) supplemented with 10\% Fetal Bovine Serum (Gibco, Life 
465 Technologies) and $1 \%$ Penicillin-Streptomycin (Corning) and maintained at $37^{\circ} \mathrm{C}$ in a humidified

466 atmosphere of $5 \% \mathrm{CO}_{2}$.

467

468 Transfection. For each affinity purification (wild-type and mutant nsp2 and controls, empty 469 vector and EGFP), 7.5 million HEK293T cells were plated per 15-cm dish and allowed to 470 recover for $20-24$ hours prior to transfection. Up to $15 \mu \mathrm{g}$ of individual Strep-tagged expression 471 constructs (normalized to $15 \mu \mathrm{g}$ with empty vector as needed) was complexed with PolyJet 472 Transfection Reagent (SignaGen Laboratories) at a 1:3 $\mu \mathrm{g}: \mu \mathrm{l}$ ratio of plasmid to transfection 473 reagent based on manufacturer's recommendations. After 40 hours, cells were dissociated at 474 room temperature using $10 \mathrm{ml}$ Dulbecco's Phosphate Buffered Saline without calcium and 475 magnesium (D-PBS) supplemented with $10 \mathrm{mM}$ EDTA for at least 5 minutes and subsequently 476 washed with $10 \mathrm{ml}$ D-PBS. Each step was followed by centrifugation at $200 \mathrm{xg}, 4^{\circ} \mathrm{C}$ for 5 477 minutes. Cell pellets were frozen on dry ice and stored at $-80^{\circ} \mathrm{C}$. At least three biological 478 replicates were independently prepared for affinity purification.

480 Affinity purification. Frozen cell pellets were thawed on ice for $15-20$ minutes and suspended 481 in $1 \mathrm{ml}$ Lysis Buffer [IP Buffer (50 mM Tris- $\mathrm{HCl}, \mathrm{pH} 7.4$ at $4^{\circ} \mathrm{C}, 150 \mathrm{mM} \mathrm{NaCl}, 1 \mathrm{mM}$ EDTA) 482 supplemented with $0.5 \%$ Nonidet P 40 Substitute (NP40; Fluka Analytical) and cOmplete mini 483 EDTA-free protease and PhosSTOP phosphatase inhibitor cocktails (Roche)]. Samples were 484 then frozen on dry ice for $10-20$ minutes and partially thawed in a $37^{\circ} \mathrm{C}$ water bath. Following 485 two freeze-thaw cycles, samples were incubated on a tube rotator for 30 minutes at $4^{\circ} \mathrm{C}$ and 486 centrifuged at $13,000 \mathrm{xg}, 4^{\circ} \mathrm{C}$ for 15 minutes to pellet debris. After reserving $50 \mu$ lysate, 487 samples were arrayed into a 96-well Deepwell plate for affinity purification on the KingFisher 488 Flex Purification System (Thermo Scientific) as follows: MagStrep "type3" beads (30 $\mu$; IBA 489 Lifesciences) were equilibrated twice with $1 \mathrm{ml}$ Wash Buffer (IP Buffer supplemented with 0.05\% 
490 NP40) and incubated with $\sim 0.95 \mathrm{ml}$ lysate for 2 hours. Beads were washed three times with 1 $491 \mathrm{ml}$ Wash Buffer, once with $1 \mathrm{ml}$ IP Buffer and then transferred to $75 \mu \mathrm{l}$ Denaturation-Reduction

492 Buffer [2 M urea, 50 mM Tris-HCl pH 8.0, 1 mM DTT) aliquoted into 96-well plates for on-bead 493 digestion (below)]. The KingFisher Flex Purification System was placed in the cold room and 494 allowed to equilibrate to $4^{\circ} \mathrm{C}$ overnight before use. All automated protocol steps were performed 495 using the slow mix speed and the following mix times: 30 seconds for equilibration/wash steps, 4962 hours for binding, and 1 minute for final bead release. Three 10 second bead collection times 497 were used between all steps.

\section{8}

499 On-bead digestion. Bead-bound proteins were incubated in Denaturation-Reduction Buffer for 50030 minutes, alkylated in the dark with $3 \mathrm{mM}$ iodoacetamide for 45 minutes and quenched with 3 $501 \mathrm{mM}$ DTT for 10 minutes. Proteins were then trypsin digested as follows: initially for 4 hours with $5021.5 \mu \mathrm{l}$ trypsin $(0.5 \mu \mathrm{g} / \mu \mathrm{l}$; Promega) and then another 2 hours with $0.5 \mu \mathrm{l}$ additional trypsin. To 503 offset evaporation during trypsin digestion, $22.5 \mu \mathrm{l} 50 \mathrm{mM}$ Tris- $\mathrm{HCl}$, pH 8.0 was added. All steps 504 were performed with constant shaking at $1,100 \mathrm{rpm}$ on a ThermoMixer $\mathrm{C}$ incubator set to $37^{\circ} \mathrm{C}$ 505 (denaturation-reduction and trypsin digest) or room temperature (alkylation and quenching). 506 Digested peptides were combined with $50 \mu \mathrm{l} 50 \mathrm{mM}$ Tris- $\mathrm{HCl}, \mathrm{pH} 8.0$ used to backwash beads 507 and acidified with trifluoroacetic acid $(0.5 \%$ final, $\mathrm{pH}<2.0)$ Acidified peptides were desalted 508 using a BioPureSPE Mini 96-Well Plate (20mg PROTO 300 C18; The Nest Group, Inc.) 509 according to standard protocols and dried in a CentriVap Concentrator (Labconco) for at least 510 two hours.

511 Mass spectrometry data acquisition and analysis. Samples were re-suspended in $4 \%$ formic 512 acid, 2\% acetonitrile solution, and separated by a reversed-phase gradient over a nanoflow C18 513 column (Dr. Maisch). Each sample was directly injected via a Easy-nLC 1200 (Thermo Fisher 514 Scientific) into a Q-Exactive Plus mass spectrometer (Thermo Fisher Scientific) and analyzed 
515 with a 75 min acquisition, with all MS1 and MS2 spectra collected in the orbitrap; data were 516 acquired using the Thermo software Xcalibur (4.2.47) and Tune (2.11 QF1 Build 3006). For all 517 acquisitions, QCloud was used to control instrument longitudinal performance during the

518 project $^{53}$. All proteomic data was searched against the human proteome (uniprot reviewed 519 sequences downloaded February 28th, 2020), EGFP sequence, and the SARS-CoV-2 protein

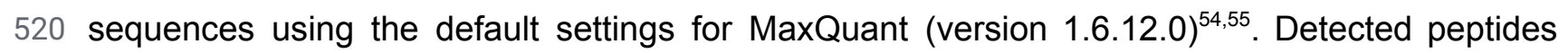
521 and proteins were filtered to $1 \%$ false discovery rate in MaxQuant.

522 Identified proteins were then subjected to protein-protein interaction scoring with both 523 SAINTexpress (version 3.6.3) ${ }^{56}$ and MiST (https://github.com/kroganlab/mist) ${ }^{57,58}$. Interactions 524 passing the master threshold (MiST score $\geq 0.6$, a SAINTexpress BFDR $\leq 0.05$ and an average 525 spectral count $\geq 2$ ) for at least one of the baits (mutants or wild-types) were kept for further 526 analysis. An "Interaction Score" was defined as the average between the MiST score and the 527 Saint Score, as previously described ${ }^{10}$. In addition, interactions were removed if their detection 528 was found to be discrepant for wild-type Nsp2 between this study and a prior study ${ }^{2}$ (difference 529 in interaction scores between studies < 0.4), further increasing the confidence of our final set of 530 interactions. Remaining interactions were separated into two groups: those interacting with 531 Nsp2 wild-type or not (average Interaction Score $>0.5$ or $<0.5$ were separated into Fig 5A-C 532 and Sup Fig 5A, respectively). For those interacting with wild-type, a quantitative statistical 533 analysis was performed (this quantitation was not possible for those not interacting with 534 wild-type Nsp2 due to the lack of detection). Specifically, prey intensities in each affinity 535 purification were normalized to the corresponding bait abundance using MSstats ${ }^{59}$ 536 (globalStandards norm). $\log _{2}$ fold changes and $\mathrm{BH}$-adjusted $\mathrm{p}$-values were calculated by 537 comparing each mutant to the wild-type from this study.

538 Scheduled parallel reaction monitoring (PRM) analysis of Nsp2 interactors. Peptides for 539 targeted MS were selected after importing the msms.txt file derived from the previously 
540 described MaxQuant search into Skyline $(\mathrm{v} 20.2 .0 .343)^{60}$. Proteotypic peptides passing an 541 Andromeda score of 0.95 were selected and manually inspected to choose precursors suitable

542 for targeted proteomics. In total 4 peptides per protein were selected for targeted analysis. For 543 WASHC4 and EIF4E2 all peptides identified by DDA were used ( 3 and 2 respectively). The 544 samples from AP-MS were acquired in Partial Reaction Monitoring mode (PRM) ${ }^{61}$ on a 545 Q-Exactive Orbitrap (Thermo Fisher) connected to a nanoLC easy 1200 (Thermo Fisher). 546 Peptides for the scheduled analysis were separated in 75 minutes using the same 547 chromatographic gradient and source parameter to the DDA samples. Precursor ion scans were 548 recorded in the Orbitrap at 70'000 resolution (at $400 \mathrm{~m} / \mathrm{z}$ ) for $100 \mathrm{~ms}$ or until the ion population 549 reached an AGC value of $1 e^{6}$. Peptides in the inclusion list were fragmented using HCD with a 550 normalized collisional energy of 27 , an isolation window of $2 \mathrm{Da}$ and a scheduled retention time 551 window of 7 minutes. Fragments were acquired in the Orbitrap at $17^{\prime} 500$ resolution (at $400 \mathrm{~m} / \mathrm{z}$ ) 552 for $100 \mathrm{~ms}$ or until reaching an AGC of $2 \mathrm{e}^{5}$. Loop count was set to 20 . For data analysis, the 553 PRM data was searched with MaxQuant using a FASTA file containing only the target proteins 554 and default settings. The msms.txt was imported into Skyline using the 'import peptide search' 555 option and setting the search type to targeted. To import the files, the following transition 556 settings were used: The MS1 filter was disabled, ion types were set to y and $b$ and MS/MS 557 settings were set to Orbitrap as mass analyzer, type as targeted and resolution of 17500 (at 400 $558 \mathrm{~m} / \mathrm{z}$ ). Peptides with poor coeluting fragments (dotp lower than 0.9) were removed. WASHC4 559 peptides did not pass this quality control criterion and thus WASHC4 was not considered for 560 further analysis. After import, peak boundaries were manually corrected and noisy transitions 561 were removed. The resulting data was exported at the transition level and transitions missing in 562 more than half of the samples were removed to ensure robust quantitation. The resulting 563 transitions were summed up per peptide and then the experiment was mean centered using the 564 average peptide level for the bait protein (using SARS-Cov-2 Nsp2 quantity for SARS-CoV-2 565 mutants). Normalized peptide-level abundances were averaged to reach protein level and $\log _{2}$ 
566 transformed. The fold change and $\mathrm{BH}$-adjusted $\mathrm{p}$-values for condition were calculated against

567 the wild-type Nsp2.

568 Acknowledgments.

569 The authors acknowledge their partners and families for support in childcare and other matters

570 during this time. We thank D. Mullins for helpful discussions and V. Ramani for providing critical

571 feedback on the manuscript. The structural biology portion of this work was performed by the

572 QCRG (Qquantitative Biosciences Institute $\underline{\text { Coronavirus }}$ Research Group) Structural Biology

573 Consortium. Listed below are the contributing members of the consortium listed by teams in

574 order of team relevance to the published work. Within each team the team leads are italicized

575 (responsible for organization of each team, and for the experimental design utilized within each

576 team), then the rest of team members are listed alphabetically. Bacterial expression team:

577 Amy Diallo, Meghna Gupta, Erron W. Titus, Jen Chen, Loan Doan, Sebastian Flores, Mingliang

578 Jin, Huong T. Kratochvil, Victor L. Lam, Yang Li, Megan Lo, Gregory E. Merz, Joana Paulino,

579 Aye C. Thwin, Zanlin Yu, Fengbo Zhou, Yang Zhang. Protein purification team: Michelle

580 Moritz, Tristan W. Owens, Sergei Pourmal, Caleigh M. Azumaya, Cynthia M. Chio, Bryan

581 Faust, Meghna Gupta, Kate Kim, Joana Paulino, Komal Ishwar Pawar, Jessica K. Peters,

582 Kaitlin Schaefer, Ursula Schulze-Gahmen, Tsz Kin Martin Tsui. CryoEM grid

583 freezing/collection team: Caleigh M. Azumaya, Axel F. Brilot, Gregory E. Merz, Cristina

584 Puchades, Alexandrea N. Rizo, Ming Sun, Julian R. Braxton, Meghna Gupta, Fei Li, Kyle E.

585 Lopez, Arthur Melo, Gregory E. Merz, Frank R. Moss III, Joana Paulino, Thomas H. Pospiech, 586 Jr., Sergei Pourmal, Amber M. Smith, Paul V. Thomas, Feng Wang, Zanlin Yu. CryoEM data 587 processing team: Axel F. Brilot, Miles Sasha Dickinson, Gregory E. Merz, Henry C. Nguyen,

588 Alexandrea N. Rizo, Daniel Asarnow, Julian R. Braxton, Melody G. Campbell, Cynthia M. Chio,

589 Un Seng Chio, Devan Diwanji, Bryan Faust, Soumya Govinda Remesh, Meghna Gupta, Nick

590 Hoppe, Mingliang Jin, Fei Li, Junrui Li, Yanxin Liu, Adamo Mancino, Melissa Mendez, Joana

591 Paulino, Thomas H. Pospiech, Jr., Sergei Pourmal, Smriti Sangwan, Raphael Trenker, Donovan 
592 Trinidad, Eric Tse, Kaihua Zhang, Fengbo Zhou. Mammalian cell expression team: Christian

593 Billesboelle, Melody G. Campbell, Devan Diwanji, Evelyn Hernandez, Carlos Nowotny, Amber 594 M. Smith, Jianhua Zhao, Caleigh M. Azumaya, Alisa Bowen, Nick Hoppe, Yen-Li Li, Edmond 595 Linossi, Jocelyne Lopez, Phuong Nguyen, Michael D. Paul, Cristina Puchades, Mali Safari, 596 Smriti Sangwan, Kaitlin Schaefer, Raphael Trenker, Tsz Kin Martin Tsui, Natalie Whitis.

597 Crystallography team: Nadia Herrera, Huong T. Kratochvil, Ursula Schulze-Gahmen, Iris D.

598 Young, Justin Biel, Ishan Deshpande, Xi Liu. Infrastructure team: David Bulkley, Arceli Joves, 599 Almarie Joves, Liam McKay, Mariano C. Tabios, Eric Tse. Leadership team: Oren S 600 Rosenberg, Kliment A Verba, David A Agard, Yifan Cheng, James S Fraser, Adam Frost, 601 Natalia Jura, Tanja Kortemme, Nevan J Krogan, Aashish Manglik, Daniel R. Southworth, Robert 602 M Stroud. The QCRG Structural Biology Consortium has received support from: Quantitative 603 Biosciences Institute, Defense Advance Research Projects Agency HR0011-19-2-0020 (to 604 D.A.A. and K.A.V.; B. Shoichet PI), FastGrants COVID19 grant (K.A.Verba PI), Laboratory For 605 Genomics Research (O.S. Rosenberg PI) and Laboratory For Genomics Research (R.M. Stroud $606 \mathrm{PI})$

607

\section{Author contribution statement.}

609 The following authors designed and generated protein constructs and also performed protein 610 expressions: M.G., A.D., G.E.M., D.C.D., E.H., V.L.L., Y.L., C.A.N., A.M.S., Z.Y., M.G.C., J.C., 611 L.D., Y.L., E.L., M.L., J.L., M.D.P., M.S., A.C.T., E.W.T., R.T., Y.Z., J.Z.. The following authors 612 performed protein purifications: C.M.A., M.M., S.P., T.W.O., J.K.P., U.S.G., K.K., K.I.P., K.S., 613 T.K.M.T., F.Z.. The following authors performed structural analysis (data collection by 614 crystallography or cryoEM and data processing and model building): M.G., C.M.A., S.P., G.E.M., 615 A.F.B., N.H., H.T.K., F.L., H.C.N., A.N.R., I.D.Y., D.E.A., U.S.C., M.S.D., M.J., J.L., Y.L., K.E.L., 616 A.M., F.R.M., T.H.P.Jr, C.P., S.G.R., M.S., E.T.. Graphene oxide grids were provided by F.W. and 617 Z.Y.. M.C.T. provided crucial infrastructural and organizational support. The following authors did 
618 affinity purification mass spectrometry studies: G.M.J., M.B., A.F., K.C., A.P., Y.Z.. The following 619 authors supervised or managed research: D.A.A., Y.C., J.S.F., N.J., T.K., A.M., D.R.S., R.M.S., 620 D.L.S., N.J.K., A.F., O.S.R., K.A.V.. The following authors designed and conceptualized the 621 study: M.G., C.M.A., M.M., S.P., A.D., G.E.M., M.B., L.Z.A., D.A.A., Y.C., J.S.F., N.J., T.K., A.M., 622 D.R.S., R.M.S., N.J.K., A.F., O.S.R., K.A.V.. The following drafted the initial manuscript: M.G., 623 M.M., S.P., G.E.M., G.M.J., M.B., A.F., F.L., L.Z.A., J.S.F., D.L.S., N.J.K., A.F., O.S.R., K.A.V.. All 624 authors edited the manuscript.

625

\section{Competing interests statement.}

627 J.S.F. is a founder of Keyhole Therapeutics and a shareholder of Relay Therapeutics and 628 Keyhole Therapeutics. The Fraser laboratory has received sponsored research support from 629 Relay Therapeutics. The Krogan Laboratory has received research support from Vir 630 Biotechnology and F. Hoffmann-La Roche. N.K. has consulting agreements with Maze 631 Therapeutics and Interline Therapeutics, and is a shareholder of Tenaya Therapeutics. A.F. is a 632 shareholder of Relay Therapeutics. A.F. has received sponsored research support from Relay 633 Therapeutics.

634

\section{Funding statement.}

636 This research was funded by: $\mathrm{NIH} \mathrm{NCl} \mathrm{F32CA239333} \mathrm{to} \mathrm{M.B.;} \mathrm{NIH/NCI} \mathrm{1F30CA247147} \mathrm{to}$ 637 D.C.D.; BWF 1019894 to N.H.; NIGMS K99GM138753 to H.T.K.; NIMH K99MH119591 to F.L.; 638 Damon Runyon Cancer Research Foundation postdoctoral award to T.W.O.; NIH 639 F32GM133084 to J.K.P.; NIH F32GM133129 to I.D.Y.; Alfred Benzon Foundation award to 640 C.B.B.; NIH NIGMS F32GM137463 to U.S.C.; American Heart Association \#18POST33990362 641 to Y.L.; NIH T32EB9383 to K.E.L.; Damon Runyon Cancer Research Foundation postdoctoral 642 award to C.P.; Helen Hay Whitney Foundation to K.S.; DFG GZ: TR 1668/1-1 to R.T.; Human 643 Frontier Science Program (HFSP) fellowship to K.Z.; NSF RAPID 2031205 to J.S.F.; NIH GM 
644 24485, Al095208, Al50476 to R.M.S.; FastGrants COVID-19 grant to K.A.V.; Laboratory For 645 Genomics Research grant to O.S.R. and R.M.S.; NIH (1R01Al128214, U19Al135990) to OSR; 646 NIH (P50Al150476, U19Al135990, U19Al135972, R01Al143292, R01Al120694, and 647 P01Al063302) to N.J.K; Excellence in Research Award (ERA) from the Laboratory for 648 Genomics Research (LGR), a collaboration between UCSF, UCB, and GSK (\#133122P) to 649 N.J.K; Roddenberry Foundation to N.J.K; funding from F. Hoffmann-La Roche and Vir 650 Biotechnology and gifts from QCRG philanthropic donors. This work was supported by the 651 Defense Advanced Research Projects Agency (DARPA) under Cooperative Agreement 652 \#HR0011-19-2-0020 (N.J.K, D.A.A., K.A.V.). The views, opinions, and/or findings contained in 653 this material are those of the authors and should not be interpreted as representing the official 654 views or policies of the Department of Defense or the U.S. Government. The UCSF Electron 655 Microscopy Facilities were partially funded by following grants: 1S10OD026881-01, 656 1S10RR026814-01, 1S10OD020054-01, 1S10OD021741-01.

657

658 Data availability statement.

659 The atomic coordinates and the associated cryoEM maps for both Nsp2 structures, with and 660 without zinc were deposited to the PDB and EMDB with PDB codes of XXXX and YYYY and 661 EMDB codes of XXXXX and YYYYY. The mass spectrometry proteomics data have been 662 deposited to the ProteomeXchange Consortium via the PRIDE partner repository with the 663 dataset identifier PXD025817. Reviewers may access the dataset using the following 664 credentials: Username of reviewer_pxd025817@ebi.ac.uk and password of szYFYpXQ.

665

666 
667

668 1. V'kovski, P., Kratzel, A., Steiner, S., Stalder, H. \& Thiel, V. Coronavirus biology and

669 replication: implications for SARS-CoV-2. Nat. Rev. Microbiol. (2020)

670 doi:10.1038/s41579-020-00468-6.

671 2. Gordon, D. E. et al. A SARS-CoV-2 protein interaction map reveals targets for drug 672 repurposing. Nature 583, 459-468 (2020).

673 3. Thoms, M. et al. Structural basis for translational shutdown and immune evasion by the 674 Nsp1 protein of SARS-CoV-2. Science 369, 1249-1255 (2020).

675 4. Miorin, L. et al. SARS-CoV-2 Orf6 hijacks Nup98 to block STAT nuclear import and 676 antagonize interferon signaling. Proc. Natl. Acad. Sci. U. S. A. 117, 28344-28354 (2020).

677 5. García-Sastre, A. Ten Strategies of Interferon Evasion by Viruses. Cell Host Microbe 22, 678 176-184 (2017).

679 6. Graham, R. L., Sims, A. C., Brockway, S. M., Baric, R. S. \& Denison, M. R. The nsp2 680 replicase proteins of murine hepatitis virus and severe acute respiratory syndrome 681 coronavirus are dispensable for viral replication. J. Virol. 79, 13399-13411 (2005).

682 7. Gadlage, M. J., Graham, R. L. \& Denison, M. R. Murine coronaviruses encoding nsp2 at 683 different genomic loci have altered replication, protein expression, and localization. J. Virol.

$684 \quad 82,11964-11969$ (2008).

685 8. Cornillez-Ty, C. T., Liao, L., Yates, J. R., 3rd, Kuhn, P. \& Buchmeier, M. J. Severe acute 686 respiratory syndrome coronavirus nonstructural protein 2 interacts with a host protein 687 complex involved in mitochondrial biogenesis and intracellular signaling. J. Virol. 83, $688 \quad 10314-10318$ (2009).

689 9. von Brunn, A. et al. Analysis of intraviral protein-protein interactions of the SARS 690 coronavirus ORFeome. PLoS One 2, e459 (2007).

691 10. Gordon, D. E. et al. Comparative host-coronavirus protein interaction networks reveal 692 pan-viral disease mechanisms. Science 370, (2020). 
693 11. Davies, J. P., Almasy, K. M., McDonald, E. F. \& Plate, L. Comparative multiplexed

694 interactomics of SARS-CoV-2 and homologous coronavirus non-structural proteins

695 identifies unique and shared host-cell dependencies. bioRxiv (2020)

696 doi:10.1101/2020.07.13.201517.

697 12. V'kovski, P. et al. Determination of host proteins composing the microenvironment of 698 coronavirus replicase complexes by proximity-labeling. Elife 8, (2019).

699 13. Zhao, J., Zhai, X. \& Zhou, J. Snapshot of the evolution and mutation patterns of

700 SARS-CoV-2. bioRxiv (2020) doi:10.1101/2020.07.04.187435.

701 14. Deng, X. et al. Transmission, infectivity, and antibody neutralization of an emerging

702 SARS-CoV-2 variant in California carrying a L452R spike protein mutation.

703 doi:10.1101/2021.03.07.21252647.

704 15. Hodcroft, E. B. et al. Emergence in late 2020 of multiple lineages of SARS-CoV-2 Spike

705

706

707

708

709

710 protein variants affecting amino acid position 677. medRxiv (2021) doi:10.1101/2021.02.12.21251658.

16. Hoffmann, H.-H. et al. Functional interrogation of a SARS-CoV-2 host protein interactome identifies unique and shared coronavirus host factors. Cell Host Microbe (2020) doi:10.1016/j.chom.2020.12.009.

17. Punjani, A., Rubinstein, J. L., Fleet, D. J. \& Brubaker, M. A. cryoSPARC: algorithms for rapid unsupervised cryo-EM structure determination. Nat. Methods 14, 290-296 (2017).

18. Punjani, A., Zhang, H. \& Fleet, D. J. Non-uniform refinement: adaptive regularization improves single-particle cryo-EM reconstruction. Nat. Methods 17, 1214-1221 (2020).

19. Zivanov, J. et al. New tools for automated high-resolution cryo-EM structure determination in RELION-3. Elife 7, (2018).

20. Pfab, J., Phan, N. M. \& Si, D. DeepTracer for fast de novo cryo-EM protein structure modeling and special studies on CoV-related complexes. Proc. Natl. Acad. Sci. U. S. A. 118, (2021). 
719 21. Pfab, J. \& Si, D. DeepTracer: Predicting Backbone Atomic Structure from High Resolution

720 Cryo-EM Density Maps of Protein Complexes. doi:10.1101/2020.02.12.946772.

721 22. Emsley, P., Lohkamp, B., Scott, W. G. \& Cowtan, K. Features and development of Coot.

722 Acta Crystallogr. D Biol. Crystallogr. 66, 486-501 (2010).

723 23. Croll, T. I. ISOLDE: a physically realistic environment for model building into low-resolution 724 electron-density maps. Acta Crystallogr D Struct Biol 74, 519-530 (2018).

725 24. Liebschner, D. et al. Macromolecular structure determination using X-rays, neutrons and 726 electrons: recent developments in Phenix. Acta Crystallogr D Struct Biol 75, 861-877 727 (2019).

728 25. Wang, R. Y.-R. et al. Automated structure refinement of macromolecular assemblies from 729 cryo-EM maps using Rosetta. Elife 5, (2016).

730 26. Yu, K. et al. Purification, crystallization and preliminary X-ray analysis of nonstructural 731 protein 2 (nsp2) from avian infectious bronchitis virus. Acta Crystallogr. Sect. F Struct. Biol. 732 Cryst. Commun. 68, 716-719 (2012).

733 27. Callaway, E. 'It will change everything': DeepMind's Al makes gigantic leap in solving 734 protein structures. Nature vol. 588 203-204 (2020).

735 28. Computational predictions of protein structures associated with COVID-19.

$736 \mathrm{https} / / /$ deepmind.com/research/open-source/computational-predictions-of-protein-structures 737 -associated-with-COVID-19.

738 29. Hahn, S. \& Roberts, S. The zinc ribbon domains of the general transcription factors TFIIB 739 and Brf: conserved functional surfaces but different roles in transcription initiation. Genes 740 Dev. 14, 719-730 (2000).

741 30. Hagemeijer, M. C. et al. Dynamics of Coronavirus Replication-Transcription Complexes. 742 Journal of Virology vol. 84 2134-2149 (2010).

743 31. Li, Z., Jaroszewski, L., Iyer, M., Sedova, M. \& Godzik, A. FATCAT 2.0: towards a better 744 understanding of the structural diversity of proteins. Nucleic Acids Res. 48, W60-W64 
(2020).

746

747

748

749

750

751

752

753

754

755

756

757

758

759

760

761

762

763

764

765

766

767

768

769

770

32. Holm, L. DALI and the persistence of protein shape. Protein Sci. 29, 128-140 (2020).

33. Dashboard. http://covid19.datamonkey.org/.

34. Hickey, K. L. et al. GIGYF2 and 4EHP Inhibit Translation Initiation of Defective Messenger RNAs to Assist Ribosome-Associated Quality Control. Mol. Cell 79, 950-962.e6 (2020).

35. Flower, T. G. \& Hurley, J. H. Crystallographic molecular replacement using an in silico-generated search model of SARS-CoV-2 ORF8. doi:10.1101/2021.01.05.425441.

36. Viklund, I.-M. et al. WAFL, a new protein involved in regulation of early endocytic transport at the intersection of actin and microtubule dynamics. Exp. Cell Res. 315, 1040-1052 (2009).

37. Derivery, E. et al. The Arp2/3 activator WASH controls the fission of endosomes through a large multiprotein complex. Dev. Cell 17, 712-723 (2009).

38. Carnell, M. et al. Actin polymerization driven by WASH causes V-ATPase retrieval and vesicle neutralization before exocytosis. J. Cell Biol. 193, 831-839 (2011).

39. Dilucca, M., Forcelloni, S., Georgakilas, A. G., Giansanti, A. \& Pavlopoulou, A. Codon Usage and Phenotypic Divergences of SARS-CoV-2 Genes. Viruses 12, (2020).

40. Alonso, A. M. \& Diambra, L. SARS-CoV-2 Codon Usage Bias Downregulates Host Expressed Genes With Similar Codon Usage. Front Cell Dev Biol 8, 831 (2020).

41. Tollenaere, M. A. X. et al. GIGYF1/2-Driven Cooperation between ZNF598 and TTP in Posttranscriptional Regulation of Inflammatory Signaling. Cell Rep. 26, 3511-3521.e4 (2019).

42. Simmonds, P. Rampant $\mathrm{C} \rightarrow \mathrm{U}$ Hypermutation in the Genomes of SARS-CoV-2 and Other Coronaviruses: Causes and Consequences for Their Short- and Long-Term Evolutionary Trajectories. mSphere vol. 5 (2020).

43. Pohl, M. O. et al. Distinct Phenotypes of SARS-CoV-2 Isolates Reveal Viral Traits Critical for Replication in Primary Human Respiratory Cells. doi:10.1101/2020.10.22.350207. 
44. Zheng, S. Q. et al. MotionCor2: anisotropic correction of beam-induced motion for improved cryo-electron microscopy. Nat. Methods 14, 331-332 (2017).

45. Kucukelbir, A., Sigworth, F. J. \& Tagare, H. D. Quantifying the local resolution of cryo-EM density maps. Nat. Methods 11, 63-65 (2014).

46. Tan, Y. Z. et al. Addressing preferred specimen orientation in single-particle cryo-EM through tilting. Nat. Methods 14, 793-796 (2017).

47. Pettersen, E. F. et al. UCSF ChimeraX: Structure visualization for researchers, educators, and developers. Protein Sci. 30, 70-82 (2021).

48. Goddard, T. D. et al. UCSF ChimeraX: Meeting modern challenges in visualization and analysis. Protein Sci. 27, 14-25 (2018).

49. Waterhouse, A. M., Procter, J. B., Martin, D. M. A., Clamp, M. \& Barton, G. J. Jalview Version 2--a multiple sequence alignment editor and analysis workbench. Bioinformatics 25, 1189-1191 (2009).

50. Katoh, K. \& Standley, D. M. MAFFT multiple sequence alignment software version 7: improvements in performance and usability. Mol. Biol. Evol. 30, 772-780 (2013).

51. Pettersen, E. F. et al. UCSF Chimera--a visualization system for exploratory research and analysis. J. Comput. Chem. 25, 1605-1612 (2004).

52. Brown, N. P., Leroy, C. \& Sander, C. MView: a web-compatible database search or multiple alignment viewer. Bioinformatics vol. 14 380-381 (1998).

53. Chiva, C. et al. QCloud: A cloud-based quality control system for mass spectrometry-based proteomics laboratories. PLoS One 13, e0189209 (2018).

54. Cox, J. \& Mann, M. MaxQuant enables high peptide identification rates, individualized p.p.b.-range mass accuracies and proteome-wide protein quantification. Nat. Biotechnol. 26, 1367-1372 (2008).

55. Cox, J. et al. Accurate Proteome-wide Label-free Quantification by Delayed Normalization and Maximal Peptide Ratio Extraction, Termed MaxLFQ. Mol. Cell. Proteomics 13, 
56. Teo, G. et al. SAINTexpress: improvements and additional features in Significance Analysis of INTeractome software. J. Proteomics 100, 37-43 (2014).

800

57. Jäger, S. et al. Global landscape of HIV-human protein complexes. Nature 481, 365-370 (2011).

802

58. Verschueren, E. et al. Scoring Large-Scale Affinity Purification Mass Spectrometry Datasets with MiST. Curr. Protoc. Bioinformatics 49, 8.19.1-16 (2015).

59. Choi, M. et al. MSstats: an R package for statistical analysis of quantitative mass spectrometry-based proteomic experiments. Bioinformatics 30, 2524-2526 (2014).

60. MacLean, B. et al. Skyline: an open source document editor for creating and analyzing targeted proteomics experiments. Bioinformatics 26, 966-968 (2010).

61. Peterson, A. C., Russell, J. D. ., Bailey, D. J., Westphall, M. S. \& Coon, J. J. Parallel reaction monitoring for high resolution and high mass accuracy quantitative, targeted proteomics. Mol. Cell. Proteomics 11, 1475-1488 (2012). 
814 Figure 1.
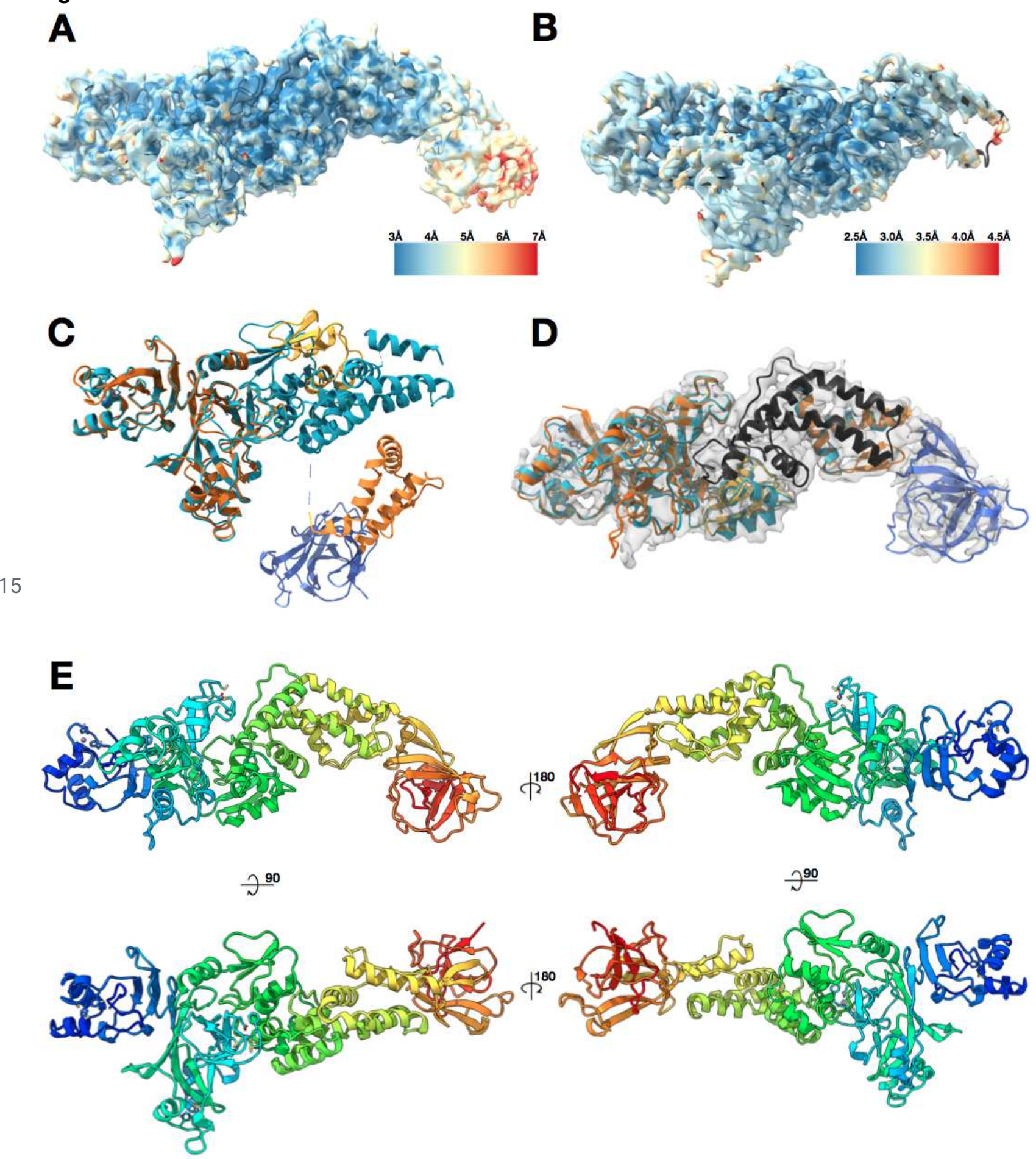

816 Figure 1. Nsp2 cryo-EM density and model overview.

817 (A) $3.8 \AA$ cryo-EM map of Nsp2 colored by local resolution showing the extra density at the 818 C-terminus (B) $3.2 \AA$ cryo-EM map of Nsp2 colored by local resolution with the resulting model 
819 in ribbon (C) Most up to date AlphaFold2 Nsp2 model (multicolored) was aligned to the 820 experimentally built Nsp2 model shown in cyan ribbon. The missing 93 amino acids from the

821 latest AlphaFold2 prediction are indicated by a dashed line. (D) AlphaFold2 Nsp2 predicted 822 model (same as $\mathbf{C}$ ) was broken into 4 regions and then individually aligned to the experimentally 823 built model (domains segmented from the AlphaFold2 prediction are in shades of orange, 824 experimental model is in cyan, in black is the region missing from the AlphaFold2 prediction but 825 built into the experimental model, in blue is the C-terminal domain as predicted by AlphaFold2 826 and fit into the $3.8 \AA$ cryo-EM map) (E) The resulting full length Nsp2 structure depicted as 827 ribbon and colored as rainbow, blue for $\mathrm{N}$ terminus to red for $\mathrm{C}$-terminus. 

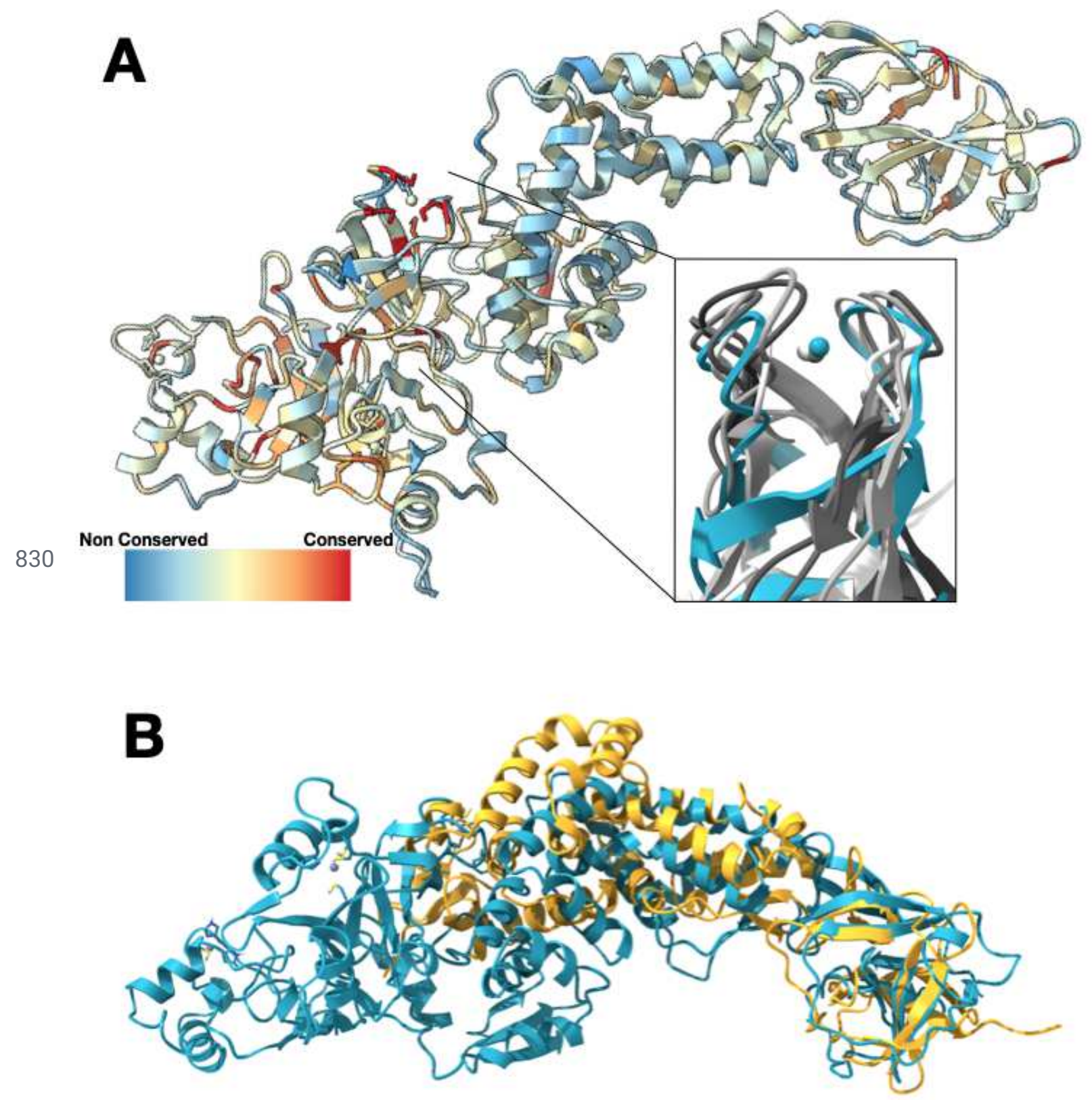

831

Figure 2. Nsp2 has a conserved zinc binding motif but otherwise shows low conservation.

834 (A) Nsp2 structure depicted as ribbon and colored by conservation (see methods for details).

835 The four cysteines show the highest conservation and are indicated in red. The magnified insert 836 shows the zinc ribbon motif of Nsp2 in cyan aligned to zinc ribbon motifs from structurally similar 837 structures in the PDB in shades of gray (PDBs: 1JJ2, 5XON, 1QUP, 4C2M). (B) Structure of IBV 
838 Nsp2 (PDB:3LD1, yellow ribbon) aligns well to the C-terminal region of SARS-CoV-2 Nsp2 839 (cyan) even though it has less than $10 \%$ sequence identity.

840 


\section{Figure 3.}

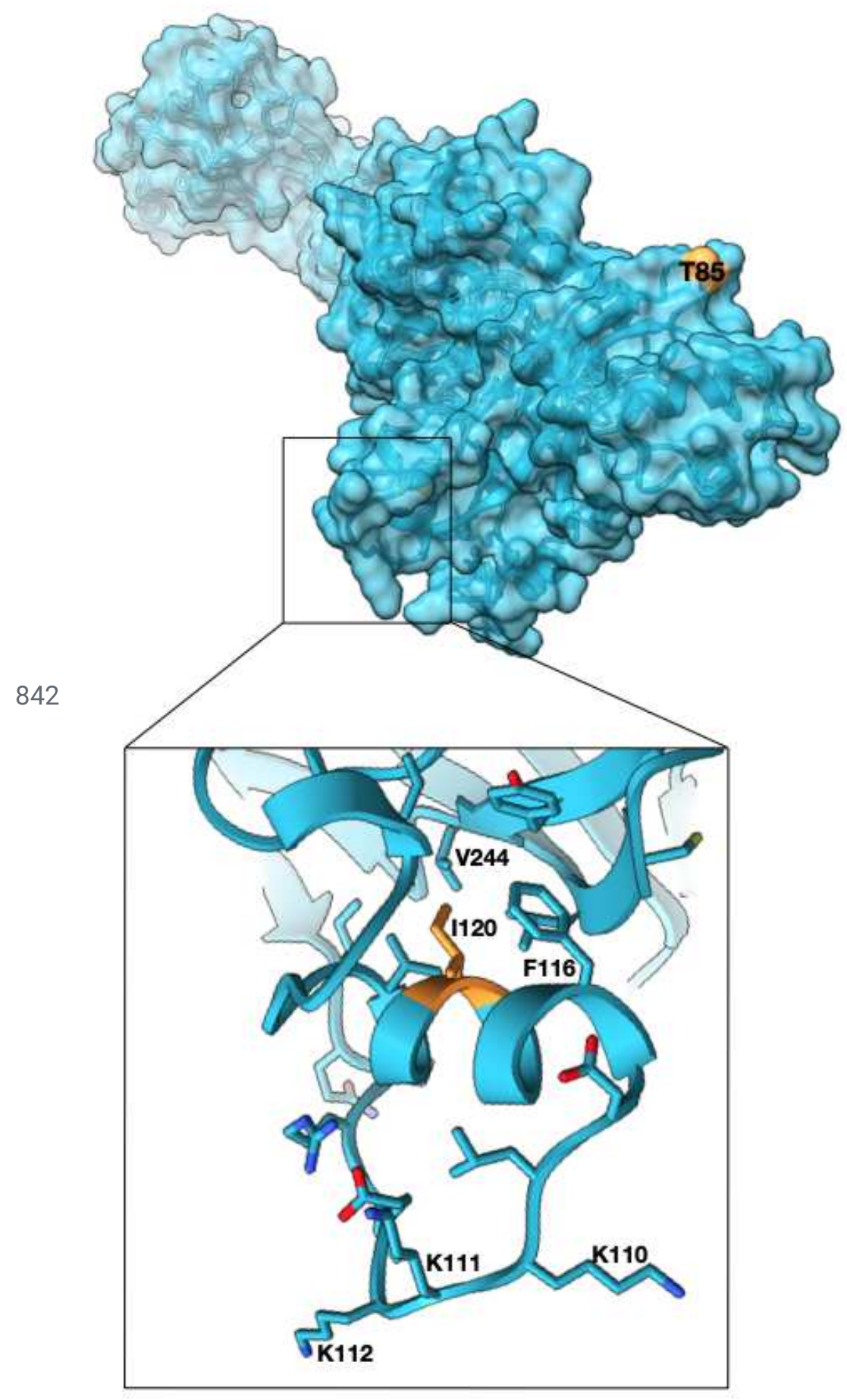

843 Figure 3. High frequency mutations in Nsp2 may provide host specific advantages.

844 T85I mutation which is present in $13 \%$ of all the SARS-CoV-2 sequences is at the surface and

845 may mediate host specific protein-protein interactions (Nsp2 surface in cyan, T85 in orange).

846 Another mutated site, 1120 points into a hydrophobic core stabilizing a small helix which is 
847 attached to a highly positively charged surface loop. Phe substitution at the site may further 848 stabilize the helix. (zoomed panel, I120 in orange).

849 


\section{Figure 4.}
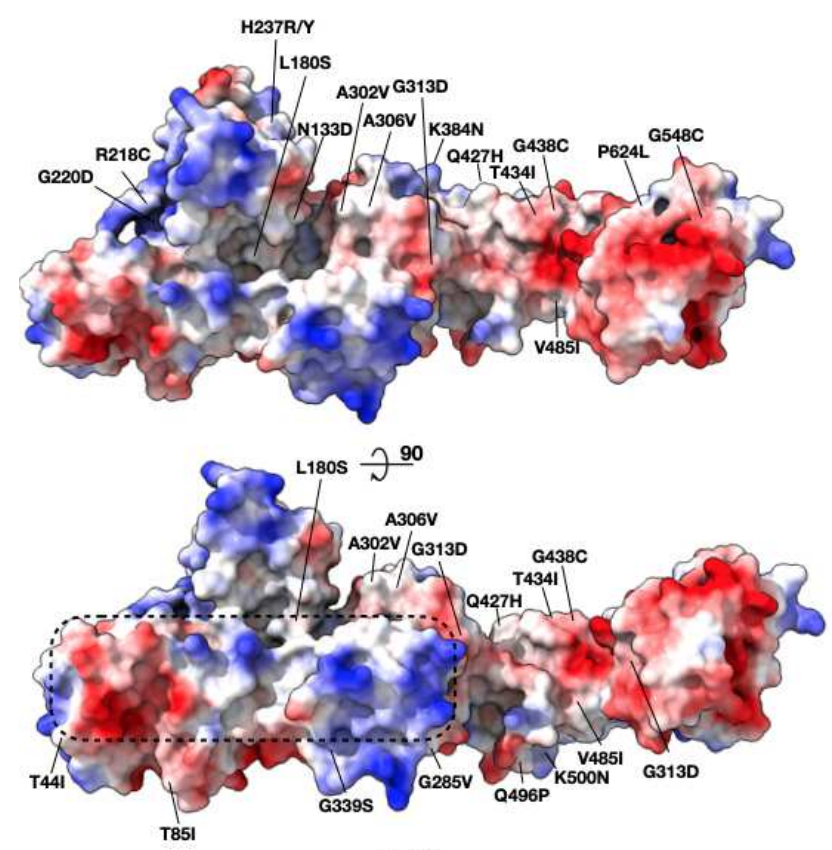

851
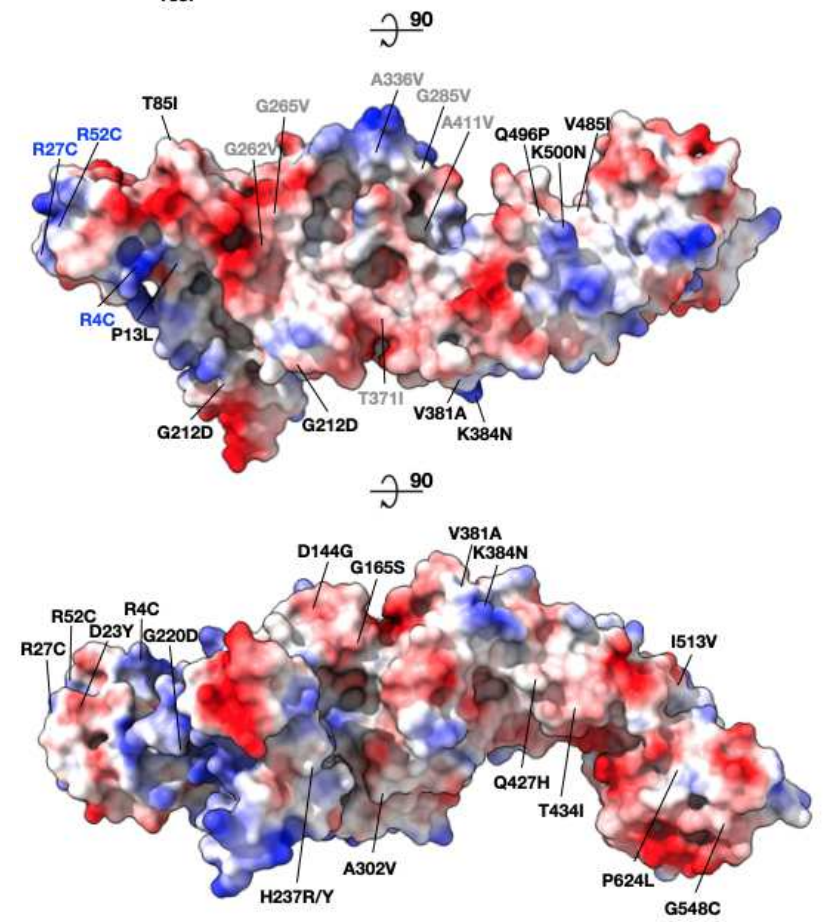

853 Figure 4. Mapping surface mutations in SARS-CoV-2 Nsp2 shows both potentially

854 constrained surfaces and rapidly changing regions.

855 All the positively selected mutations on Nsp2 mapped to the protein surface are colored by the

856 surface charge. The region that is less susceptible to mutations is inscribed in a dashed 
857 rectangle. Residues that became less charged at the N-terminus are marked in blue (cluster 1).

858 Residues that became more hydrophobic are marked in gray (cluster 2).

859 
860 Figure 5.

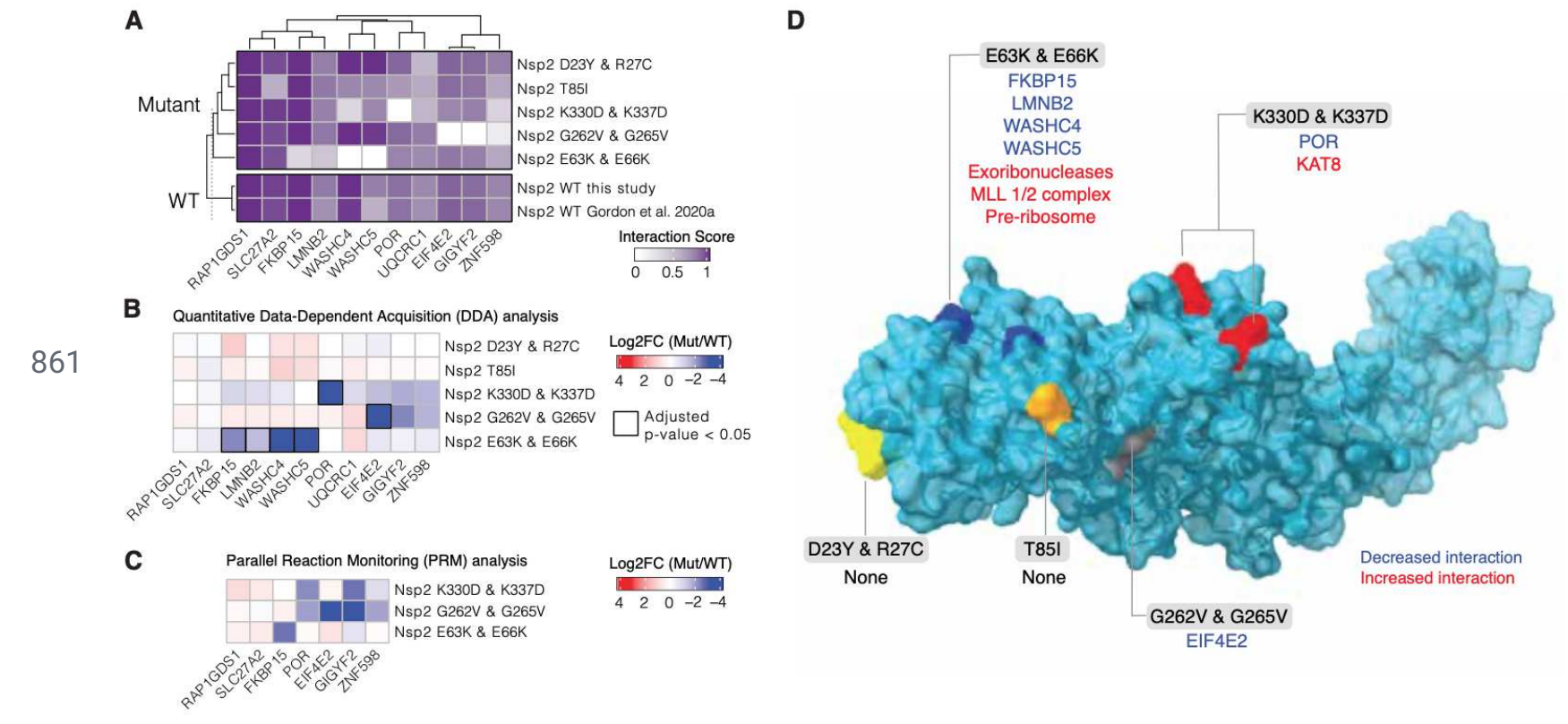

862 Figure 5. Nsp2 possesses multiple interaction surfaces for host proteins.

863 (A) Interaction scores (average between MiST and Saint Scores) for human proteins ("preys")

864 deemed high-confidence interactions in at least one affinity purification ("bait") mass

865 spectrometry assay and detected to interact with both the wild-type Nsp2 in this study and in

866 Gordon et al (2020a). Interaction scores range from zero to one, one being the most

867 high-confidence. (B) Quantitative statistical analysis of data-dependent acquisition (DDA) mass

868 spectrometry data using MSstats for interactions selected and depicted in A. Prey intensities

869 were normalized by bait expression abundance. $\log _{2}$ fold changes and $\mathrm{BH}$-adjusted $\mathrm{p}$-values

870 were calculated by comparing each mutant to the wild-type from this study. Square black

871 outlines depict adjusted p-values < 0.05 . (C) Parallel reaction monitoring (PRM) analysis of

872 select preys from B for mutants found to possess significantly-changed interactions (adjusted

873 p-value < 0.05). (D) Nsp2 structure depicted as surface (light blue) with the mutations

874 considered in this study depicted on the surface: E63K/E66K (dark blue), K330D/K337D (red),

875 D23Y/R27C (yellow), T85I (orange), and G262V/G265V (grey). Lost interactions (adjusted

876 p-value < 0.05) from data-dependent acquisition global proteomics analysis (DDA) from B

877 depicted in blue and gained protein complexes depicted in red (see Sup Fig 4) 


\section{Supplementary Files}

This is a list of supplementary files associated with this preprint. Click to download.

- D1000254629valreportfullP1.pdf

- D1000253535valreportfullP1.pdf

- SupplementaryFiles.pdf

- nreditorialpolicychecklistKVfilled.pdf

- TableS2.xIsx 